\title{
Gap Junction Dysfunction in the Prefrontal Cortex Induces Depressive-Like Behaviors in Rats
}

\author{
Jian-Dong Sun', Yan Liu', Yu-He Yuan', Jing Li' and Nai-Hong Chen*,' \\ 'State Key Laboratory of Bioactive Substances and Functions of Natural Medicines, Department of Pharmacology, Institute of Materia Medica, \\ Chinese Academy of Medical Sciences and Peking Union Medical College, Beijing, China
}

\begin{abstract}
Growing evidence has implicated glial anomalies in the pathophysiology of major depression disorder (MDD). Gap junctional communication is a main determinant of astrocytic function. However, it is unclear whether gap junction dysfunction is involved in MDD development. This study investigates changes in the function of astrocyte gap junction occurring in the rat prefrontal cortex (PFC) after chronic unpredictable stress (CUS), a rodent model of depression. Animals exposed to CUS and showing behavioral deficits in sucrose preference test (SPT) and novelty suppressed feeding test (NSFT) exhibited significant decreases in diffusion of gap junction channelpermeable dye and expression of connexin 43 (Cx43), a major component of astrocyte gap junction, and abnormal gap junctional ultrastructure in the PFC. Furthermore, we analyzed the effects of typical antidepressants fluoxetine and duloxetine and glucocorticoid receptor (GR) antagonist mifepristone on CUS-induced gap junctional dysfunction and depressive-like behaviors. The cellular and behavioral alterations induced by CUS were reversed and/or blocked by treatment with typical antidepressants or mifepristone, indicating that the mechanism of their antidepressant action may involve the amelioration of gap junction dysfunction and the cellular changes may be related to GR activation. We then investigated the effects of pharmacological gap junction blockade in the PFC on depressive-like behaviors. The results demonstrate that carbenoxolone (CBX) infusions induced anhedonia in SPT, and anxiety in NSFT, and Cx43 mimetic peptides Gap27 and Gap26 also induced anhedonia, a core symptom of depression. Together, this study supports the hypothesis that gap junction dysfunction contributes to the pathophysiology of depression.

Neuropsychopharmacology (2012) 37, I305-1320; doi:10.1038/npp.2011.319; published online 21 December 201।
\end{abstract}

Keywords: gap junction; depression; connexin 43; chronic unpredictable stress; antidepressant; glucocorticoid receptor

\section{INTRODUCTION}

Major depressive disorder (MDD) is a common and disabling illness affecting a rising percentage of the world's population. Growing evidence indicates that glial elements are involved in the neuropathology of several neuropsychiatric illnesses including MDD (Rajkowska and Miguel-Hidalgo, 2007). Post-mortem studies of tissues from patients with MDD describe a reduced number and an altered morphology of glial cells in several brain regions, in particular, the prefrontal cortex (PFC) (Ongur et al, 1998; Rajkowska et al, 1999; Cotter et al, 2001, 2002). Recent studies provide evidence that stress exposure may be related to some of the reported glial cell pathology by demonstrating that animals exposed to chronic stress have a decreased glial density in the hippocampus (Czeh et al, 2006) and a

\footnotetext{
*Correspondence: Professor N-H Chen, Department of Pharmacology, Institute of Materia Medica, Chinese Academy of Medical Sciences and Peking Union Medical College, I Xiannongtan Street, Xuanwu District, Beijing 100050, China, Tel: + 8610 63165177, Fax: + 8610 63165177, E-mail: chennh@imm.ac.cn

Received 28 July 2011; revised 23 November 201।; accepted 25 November 2011
}

reduced density of astrocytes in the prelimbic region of PFC (prelimbic cortex (PLC); Banasr and Duman, 2008). Altered number and morphology of glial cells may be the cause or consequence of glial dysfunction, and the lower densities of glial cells suggest that deficient or compromised glial function in the PFC may contribute to the symptoms of depression.

Glia and, in particular, astrocytes are organized as networks and communicate through specialized channels, the so-called gap junctions (Giaume et al, 2010). Gap junctional channels (GJCs) are composed of connexin (Cx) proteins. There are a wide variety of $\mathrm{Cxs}$ synthesized in the nervous system, and it is still a matter of controversy which Cxs are present in specific cell types. At least one, Cx43, is mostly acknowledged to be synthesized in astrocytes (Dermietzel et al, 1991; Giaume et al, 1991). GJCs are permeable to endogenous bioactive cytoplasmic molecules, and therefore the GJC-based astrocytic syncytium provides homeostatic and metabolic support likely essential for physiological neuronal function (Kimelberg, 2007). Hereby, we hypothesize that impaired gap junctional intercellular communication (GJIC) in astrocytes results in altered neuronal function in the PFC and contributes to 
malfunction in circuits involved in emotion-related subcortical centers, heavily connected with the PFC and directly involved in the pathophysiology of MDD.

To test the hypothesis, we first characterized the effects of chronic unpredictable stress (CUS), a well-documented animal model of depression (Willner, 1997, 2005), and chronic treatment with typical antidepressants including fluoxetine, a selective serotonin reuptake inhibitor (SSRI), and duloxetine, a serotonin and noradrenaline reuptake inhibitor (SNRI), on behavioral tests of depression and on the function and ultrastructure of astrocyte gap junction as well as alteration of $\mathrm{Cx} 43$ in the rat PFC. Furthermore, considering that CUS model is characterized by abnormalities in the hypothalamic-pituitary-adrenal (HPA) axis activity such as elevated corticosterone (CORT) concentrations (Ayensu et al, 1995; Holsboer and Barden, 1996; Banasr et al, 2007), and an in vitro study has shown that glucocorticoid receptor (GR) activation reduces GJIC in coupled neural progenitor cells (NPCs) (Samarasinghe et al, 2011), we analyzed the effects of mifepristone, proved to lead to the rapid amelioration of psychotic depression as GR antagonist (Belanoff et al, 2001, 2002; Simpson et al, 2005; Flores et al, 2006; DeBattista et al, 2006), on CUS-induced depressive-like behaviors and alterations of astrocyte gap junction. We then investigated the effects of pharmacological gap junction blockade in the PFC on depressive-like behaviors. For these studies, we infused gap junction blockers carbenoxolone (CBX) (Davidson and Baumgarten, 1988; Herve and Sarrouilhe, 2005), or $\mathrm{Cx} 43$ mimetic peptides Gap27 and Gap26 (Evans and Leybaert, 2007) into the PLC and measured sucrose preference known to assess anhedonia, a core symptom of depression (Willner et al, 1992), as well as the latency to feed in a novel environment, an indication of anxiety levels (Santarelli et al, 2001). We also examined the influence of $\mathrm{CBX}$ on the density of astrocytes visualized by GFAP immunoreactivity.

\section{MATERIALS AND METHODS}

\section{Animals}

Male Sprague-Dawley rats (Vital River Laboratories, Beijing, China) were housed under a 12-h light/12-h dark cycle at constant temperature $\left(22^{\circ} \mathrm{C}\right)$ with free access to food and water except when animals are subjected to stressors during the CUS procedure. Animals weighed between 250 and $300 \mathrm{~g}$ at the beginning of the experiment. All experiments were performed in accordance with the guidelines established by the National Institutes of Health for the care and use of laboratory animals and were approved by the Animal Care Committee of the Peking Union Medical College and Chinese Academy of Medical Sciences.

\section{Experimental Design}

In experiment 1 (Figure 1), rats were handled daily (home cage control, CTR) or subjected to the CUS procedure for 28 days. Animals were administered vehicle, fluoxetine, or

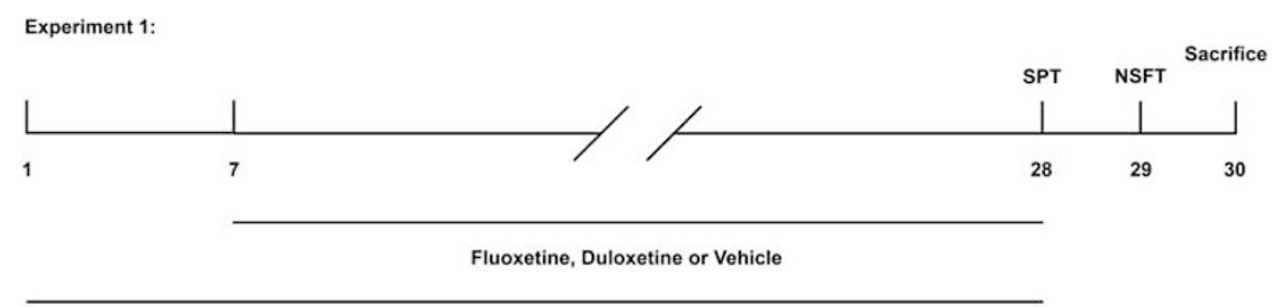

cus

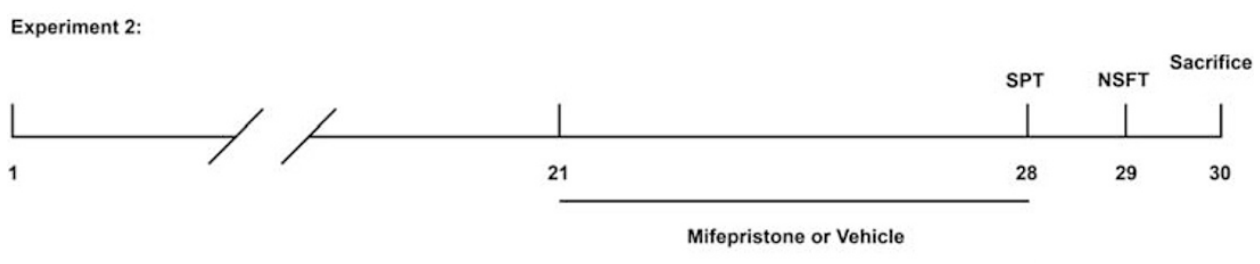

cus

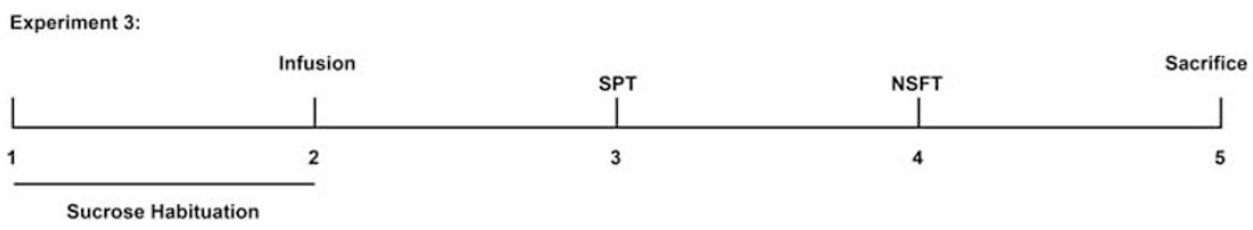

Figure I Experimental design. In experiment I, rats were handled daily (home cage control, CTR) or subjected to the chronic unpredictable stress (CUS) procedure for 28 days. Animals were administered vehicle, fluoxetine $(10 \mathrm{mg} / \mathrm{kg})$, or duloxetine $(10 \mathrm{mg} / \mathrm{kg})$ for the 21 last days of the experiment. The efficacy of CUS or antidepressants on behavioral performances of the animals in sucrose preference test (SPT) and novelty suppressed feeding test (NSFT) was measured. In experiment 2, rats were handled daily (home cage control, CTR) or subjected to the CUS procedure for 28 days. Animals were administered vehicle or mifepristone $(50 \mathrm{mg} / \mathrm{kg})$ for the 7 last days of the experiment. SPT and NSFT were also performed. In experiment 3, animals were infused with I, 10, and $100 \mathrm{mM}$ carbenoxolone (CBX) or with scrambled peptides, Gap27 and Gap26, into the prefrontal cortex (PFC), and were then subjected to the SPT and NSFT. Phosphate-buffered saline (PBS, pH 7.4) was the vehicle in the control group. 
duloxetine for the 21 last days of the experiment. The efficacy of CUS or antidepressants on behavioral performances of the animals in sucrose preference test (SPT) and novelty suppressed feeding test (NSFT) was measured on days 28 and 29. Animals were killed on day 30 , with a subset infused with Lucifer yellow (LY) for dye transfer experiment, a subset perfused for immunohistochemistry (IHC) and electron microscopy (EM), and a subset decapitated for western blotting (WB) and reverse transcriptase-PCR (RT-PCR).

Experiment 2 had similar experimental procedures with experiment 1 (Figure 1). The only difference was that animals were administered vehicle or mifepristone for the 7 last days of the CUS procedure.

Experiment 3 examined the effects of CBX on SPT and NSFT (Figure 1). Animals were infused with CBX into bilateral PLC on day 2, and were then subjected to the SPT and NSFT on days 3 and 4. Phosphate-buffered saline (PBS, $\mathrm{pH}$ 7.4) was the vehicle in the control group. Animals were then killed on day 5 , with one subset decapitated for WB, and the other subset perfused for IHC. The same protocol was also utilized to examine the effects of Gap27 and Gap26 on SPT.

\section{CUS Procedure and Drug Treatments}

CUS is an experimental procedure in which animals are exposed to a variable sequence of mild and unpredictable stressors, 2 per day for 28 days. The exact stressors and sequence used are shown in Table 1, adapted from previous studies (Banasr et al, 2007; Wu et al, 2007). For experiment 1 , animals were administered fluoxetine or duloxetine ( $10 \mathrm{mg} / \mathrm{kg}$, i.g., Lilly, Indianapolis, IN) daily for 21 days (Molteni et al, 2009; Zhang et al, 2010) and for experiment 2, animals were administered mifepristone $(50 \mathrm{mg} / \mathrm{kg}$, i.g., Beijing Zizhu Pharmaceutical Company, Beijing, China) daily for 7 days (Oomen et al, 2007).

\section{Surgery and Gap Junction Blocker Infusions}

Animals were anesthetized using chloral hydrate $(400 \mathrm{mg} / \mathrm{kg}$, i.p.) and attached to a stereotaxic apparatus. The needle of a $5 \mu$ syringe was lowered once at each cerebral hemisphere into the PLC (coordinates $(\mathrm{mm})$ : anteroposterior +3.2 ,

Table I Chronic Unpredictable Stress Procedure

\begin{tabular}{|c|c|}
\hline Day & Stressors \\
\hline Monday & Restraint $4 \mathrm{~h} ; 5 \mathrm{~min}$ hot stress in oven at $42^{\circ} \mathrm{C}$ \\
\hline Tuesday & $\begin{array}{l}\text { Food deprivation } 24 \mathrm{~h} \text {; wet bedding overnight }(200 \mathrm{ml} \text { water } \\
\text { spilled onto } 100 \mathrm{~g} \text { sawdust bedding) }\end{array}$ \\
\hline Wednesday & Isolation $24 \mathrm{~h}$ (one rat per cage); light on overnight \\
\hline Thursday & $\begin{array}{l}\text { Isolation } 24 \mathrm{~h} \text {; reversal of the light/dark cycle (light off } 12 \mathrm{~h} \\
\text { and light on overnight) }\end{array}$ \\
\hline Friday & Tail pinch I min; crowding overnight (8 rats per metabolic cage) \\
\hline Saturday & Shaker stress I h (I 60 r.p.m.); cage tilt $45^{\circ}$ overnight \\
\hline Sunday & Swim stress $12^{\circ} \mathrm{C}, 10 \mathrm{~min} ; 24 \mathrm{~h}$ water deprivation \\
\hline
\end{tabular}

The rats were exposed to these stressors according to this schedule for 4 consecutive weeks. dorsolateral -0.5, depth -4 from Bregma; Paxinos and Watson, 1998). After needle placement, $1 \mu \mathrm{l}$ of a solution containing CBX (1, 10, or $100 \mathrm{mM}$; Sigma, St Louis, MO) or vehicle (PBS) was infused bilaterally at a continuous rate of $0.2 \mu \mathrm{l} / \mathrm{min}$. These doses are within the range used in previous reports (Hosseinzadeh et al, 2005; Nilsen et al, 2006; Kokarovtseva et al, 2009). After the infusion, the needle was placed for $2 \mathrm{~min}$ and then slowly retracted. For another experiment, $1 \mu \mathrm{l}$ of a solution containing scrambled peptide, Gap27, Gap26 (Anaspec, Fremont, CA), or PBS was infused. The incision in the scalp was then sutured, triple antibiotic ointment (bacitracin, neomycin sulfate, and polymyxin B sulfate) applied, and animals were returned to their individual cages.

\section{Behavioral Tests}

SPT was conducted as previously described (Banasr et al, 2007). Briefly, rats were habituated to $1 \%$ sucrose solution for $48 \mathrm{~h}$, followed by $4 \mathrm{~h}$ of water deprivation and 1-h exposure to two identical bottles filled with either sucrose solution or water. Sucrose preference was defined as the ratio of the volume of sucrose $v s$ total volume of sucrose and water consumed during the 1 -h test.

NSFT was performed after $24 \mathrm{~h}$ of food deprivation in an open field $(76.5 \mathrm{~cm} \times 76.5 \mathrm{~cm} \times 40 \mathrm{~cm})$ with a small amount of food in the center, and latency to feed was determined as previously described (Santarelli et al, 2003; Warner-Schmidt and Duman, 2007). Home cage food intake was also measured as a control.

\section{Dye Transfer}

To determine whether gap junctions in the PFC are functional, we analyzed the diffusion of GJC-permeable dye LY, infused as described above. Briefly, after needle placement, $2 \mu \mathrm{l}$ of a solution containing LY $(5 \mu \mathrm{g} / \mu \mathrm{l})$ was infused bilaterally at a continuous rate of $0.25 \mu \mathrm{l} / \mathrm{min}$ (Rouach et al, 2008). At $30 \mathrm{~min}$ after the infusion, the animals were perfused and brains sectioned $(60 \mu \mathrm{m})$ as previously reported (Theis et al, 2003). Intercellular diffusion of fluorescent molecules was captured with a laser-scanning confocal microscope (LSCM; Leica, Wetzlar, Germany) and the distance of diffusion and the number of coupled cells was analyzed with MetaMorph software (Molecular Devices).

\section{Electron Microscopy}

Processing and EM were conducted as previously described (Jasinska et al, 2006). Briefly, anesthetized animals were perfused and the brains were trimmed to produce sections (black square shown in Figure 4a). They were postfixed with $2.5 \%$ glutaraldehyde for $2 \mathrm{~h}$, washed with $0.1 \mathrm{M}$ PBS, and then exposed to $1 \%$ osmium tetraoxide for $2 \mathrm{~h}$. After several subsequent washes with water, the tissues were dehydrated with gradient alcohol and embedded in Epon resin. Randomly selected ultrathin sections were stained with uranyl acetate and lead citrate and examined using a transmission electron microscope (H-7650, HITACHI, Tokyo, Japan).

Astrocyte gap junctions are observed at the interface between two neighboring astrocytes. Therefore, for gap 
junction structure analysis, two adjacent astrocytes were first located and pictures of the two close membranes were taken. Then, at a magnification of $\times 100000$, the integrity of the structure was evaluated and the width of gap was randomly measured at six points with MetaMorph software. Four pairs of astrocytes in each rat were analyzed $(n=6$ animals).

\section{Western Blotting}

PFC region was dissected and homogenized in lysis buffer. Protein concentrations were determined by bicinchoninic acid protein assay. A total of $15 \mu \mathrm{g}$ of proteins for each sample were separated by SDS-PAGE, and then transferred to PVDF membrane (Millipore, Temecula, CA). The membrane was blocked with $3 \%$ BSA and incubated with primary antibody overnight at $4{ }^{\circ} \mathrm{C}$ (anti-Cx43, CST, Danvers, MA, 1:1000; anti- $\beta$-actin, Sigma, 1:10000) followed by horseradish peroxidase (HRP)-conjugated secondary antibody (1:5000; KPL, Gaithersburg, MD). The protein bands were detected using enhanced chemiluminescence. Densitometric analysis of immunoreactivity for each protein was conducted using Gel-Pro Analyzer software (Media Cybernetics).

\section{RT-PCR}

Total RNA was extracted from PFC using the Trizol (Invitrogen, Carlsbad, CA) method. RNA concentration was calculated from the optical density at $260 \mathrm{~nm}$. Total RNA $(1 \mu \mathrm{g})$ from each sample was reverse transcribed into cDNA and amplified by PCR. For $\mathrm{Cx} 43$, the sense primer was $5^{\prime}$-CGCTGGAGGGAAGGTGTGGC- $3^{\prime}$ and the antisense primer was $5^{\prime}$-CCGCGCTCAAGCTGAACCCA- $3^{\prime}$ to yield a PCR product of $494 \mathrm{bp}$. The sense primer $5^{\prime}$-CGGAGAC GGGGTCACCCACA- $3^{\prime}$ and the antisense primer $5^{\prime}$-AGAGA GCCTCGGGGCATCGG- $3^{\prime}$ were used to detect $\beta$-actin, which yielded a PCR product of $320 \mathrm{bp}$. PCR was carried out by the following cycle parameters after $94{ }^{\circ} \mathrm{C}$ for $4 \mathrm{~min}$ : for $\mathrm{Cx} 43,94{ }^{\circ} \mathrm{C}$ for $40 \mathrm{~s}, 60^{\circ} \mathrm{C}$ for $40 \mathrm{~s}$, and $72{ }^{\circ} \mathrm{C}$ for $40 \mathrm{~s}$; for $\beta$-actin, $94{ }^{\circ} \mathrm{C}$ for $40 \mathrm{~s}, 58^{\circ} \mathrm{C}$ for $40 \mathrm{~s}$, and $72{ }^{\circ} \mathrm{C}$ for $40 \mathrm{~s}$. After 25 cycles of amplification, a final extension step was performed at $72{ }^{\circ} \mathrm{C}$ for $10 \mathrm{~min}$. The PCR products were electrophoretically separated on $1.5 \%$ agarose gels, stained with ethidium bromide, and visualized using electrophoresis gel imaging analysis system (Kodak, Rochester, NY).

\section{IHC and Quantification}

Anesthetized animals were perfused and brains sectioned $(30 \mu \mathrm{m})$ as previously reported (Madsen et al, 2005). Freefloating sections were incubated with blocking buffer and then with rabbit anti-Cx43 antibody (CST, 1:50) or mouse anti-GFAP antibody (1:500; Dako, Glostrup, Denmark) overnight at $4{ }^{\circ} \mathrm{C}$. Sections were then labeled for $2 \mathrm{~h}$ with FITC goat anti-rabbit antibody (Sigma, 1:400) or Alexa 488-conjugated goat anti-mouse antibody (Invitrogen, $1: 100)$ in the dark. After washes, the sections were mounted on glass slides, and then coverslipped with diazobicyclooctane-containing glycerol. Finally, the slides were examined using LSCM.
Density of Cx43-immunoreactive puncta or GFAP-positive cells was measured on six sections per animal (at the coordinates indicated for each experiment) using MetaMorph software. The main principle and the procedure of the measurements were adapted from previous studies (Wu et al, 2007; Banasr and Duman, 2008). Briefly, a $0.5 \times 0.5 \mathrm{~mm}^{2}$ contour was placed over the unilateral PLC and all Cx43-immunoreactive puncta or GFAP-positive cells within the contour were counted under high magnification $(400 \times)$. The number was measured by setting the specific uniform thresholds and results are expressed as mean \pm SEM number of Cx43-immunoreactive puncta or GFAPpositive cells per $\mathrm{mm}^{2}$.

\section{Statistical Analysis}

Statistical differences were determined by analysis of variance (ANOVA, SPSS 13.0) followed by Newman-Keuls post-hoc analysis. The level of statistical significance was set at $P<0.05$.

\section{RESULTS}

\section{Antidepressants or Mifepristone Reversed Behavioral Deficits Induced by CUS}

In the present study, we used CUS, a well-documented animal model of depression that results in multiple behavioral effects, many of which are relevant to those observed clinically, most notably anhedonia (Willner, 1997, 2005). CUS-exposed rats, when compared with unstressed control rats, exhibited a reduction in the ratio of sucrose solution to total liquid consumed, an indication of anhedonia behavior (Figure 2a and c). Treatment with $10 \mathrm{mg} /$ $\mathrm{kg}$ /day fluoxetine or duloxetine in the last 21 of 28 days of CUS or with $50 \mathrm{mg} / \mathrm{kg} /$ day mifepristone in the last 7 of 28 days of CUS significantly reversed the effect of CUS on sucrose preference (Figure 2a and c; two-way ANOVA, post-hoc Newman-Keuls, for fluoxetine: stress, $\mathrm{F}_{1,44}=8.877$, $P=0.0047$; drug, $\mathrm{F}_{1,44}=4.065, \quad P=0.0499$; interaction, $\mathrm{F}_{1,44}=4.46, P=0.0404$; for duloxetine: stress, $\mathrm{F}_{1,44}=5.258$, $P=0.0267 ; \quad$ drug, $\mathrm{F}_{1,44}=4.628, \quad P=0.037$; interaction, $\mathrm{F}_{1,44}=7.074, P=0.0109$; for mifepristone: stress, $\mathrm{F}_{1,44}=$ 12.76, $P=0.0009$; drug, $\mathrm{F}_{1,44}=9.086, P=0.0043$; interaction, $\mathrm{F}_{1,44}=15.5, P=0.0003$ ). Treatment with antidepressants or mifepristone did not affect sucrose preference in nonstressed control rats (Figure $2 \mathrm{a}$ and $\mathrm{c}$ ). In addition, no difference between groups was observed in total fluid consumption for the 1-h test (data not shown).

We also confirmed that CUS exposure increased the latency to feed in a novel environment, an indication of increased anxiety levels (Figure $2 \mathrm{~b}$ and $\mathrm{d}$ ). Although behavioral performances in NSFT of CTR animals treated with antidepressants or mifepristone and CUS animals treated with antidepressants or mifepristone were not different from that of CTR group, the latency to feed significantly reduced in CUS + fluoxetine, CUS + duloxetine, or CUS + mifepristone groups compared with CUS group (Figure 2b and d; two-way ANOVA, post-hoc NewmanKeuls, for fluoxetine: stress, $\mathrm{F}_{1,44}=5.844, P=0.0198$; drug, $\mathrm{F}_{1,44}=4.083, \quad P=0.0494$; interaction, $\mathrm{F}_{1,44}=2.52$, n.s.; for duloxetine: stress, $\mathrm{F}_{1,44}=6.197, \quad P=0.0166$; drug, 

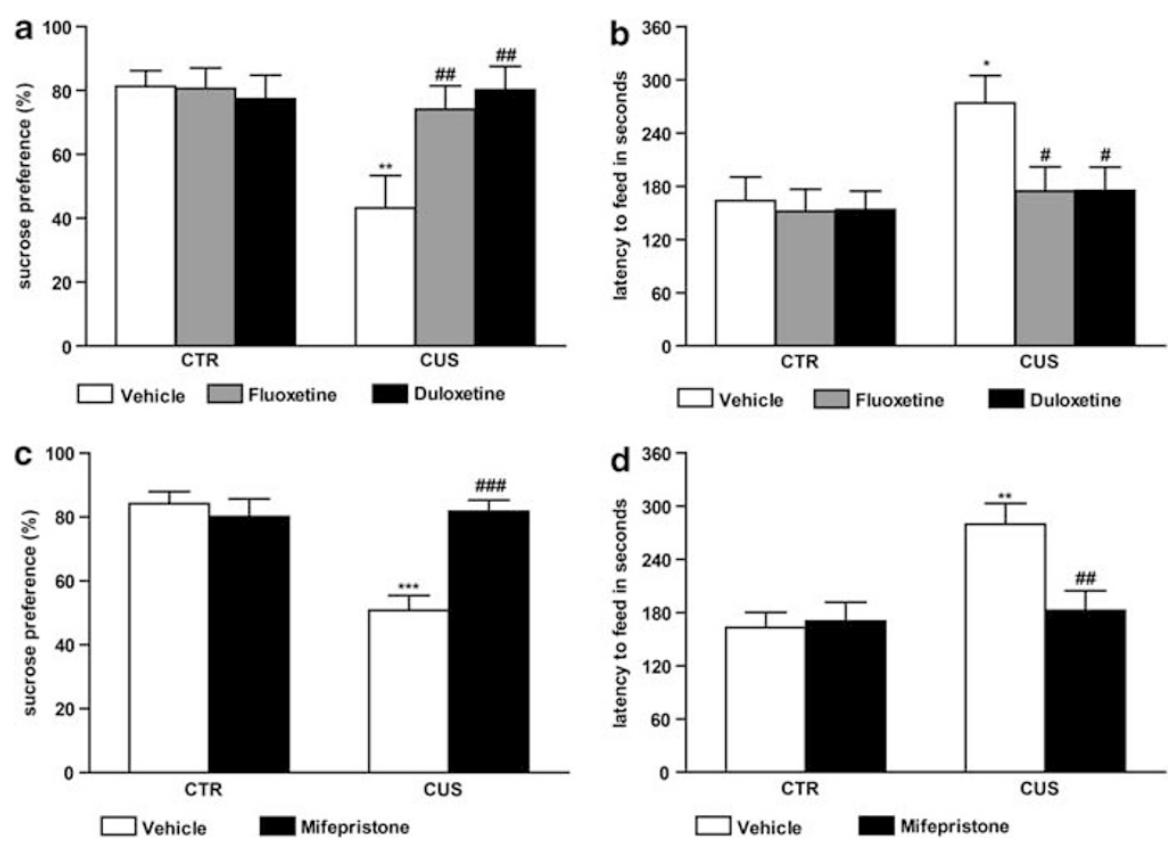

Figure 2 Antidepressants and mifepristone reversed behavioral deficits induced by chronic unpredictable stress (CUS; $n=12 /$ group). (a) Sucrose preference was decreased by 28 days of CUS exposure and was reversed by antidepressants treatment. (b) CUS animals showed a significant increase of latency to feed and this effect was reversed by antidepressants treatment. (c) Sucrose preference was decreased by 28 days of CUS exposure and was reversed by mifepristone treatment. (d) CUS animals showed a significant increase of latency to feed and this effect was reversed by mifepristone treatment. Error bars represent SEM. ${ }^{*} P<0.05$, **P $P 0.01$, **** $P<0.001$ compared with CTR and ${ }^{\#} P<0.05$, ${ }^{\# \#} P<0.0$ I, ${ }^{\# \# \#} P<0.00$ I compared with CUS, two-way analysis of the variance (ANOVA), Newman-Keuls post-hoc analysis.

$\mathrm{F}_{1,44}=4.235, P=0.0455$; interaction, $\mathrm{F}_{1,44}=2.802$, n.s.; for mifepristone: stress, $\mathrm{F}_{1,44}=9.236, P=0.004$; drug, $\mathrm{F}_{1,44}=$ 4.531, $P=0.0389$; interaction, $\left.\mathrm{F}_{1,44}=6.089, P=0.0176\right)$. In addition, there was no difference in home cage feeding conducted immediately following the NSFT (Supplementary Figure S1).

In summary, our results demonstrate that CUS induces anhedonia and anxiety and that these behavioral deficits can be reversed by treatment with antidepressants or mifepristone. In contrast to chronic (21 days) administration of typical antidepressants, the actions of mifepristone are rapid (7 days), which are consistent with previous reports (Wu et al, 2007; Zhang et al, 2010).

\section{Antidepressants or Mifepristone Reversed Intercellular Diffusion Decrease of LY Induced by CUS}

To evaluate the function of gap junctions in the PFC, we infused in the PLC the small-molecular-weight fluorescent molecule LY (Mr 457), which readily passes gap junctions. The dye transfer assay was performed simulating the procedure of the 'scrape-loading' dye transfer assay, which is used to measure the biological activity/connectivity of gap junctions in vitro (Xia et al, 2010). After $30 \mathrm{~min}$ of dye diffusion, intercellular coupling was observed. Most of the coupled cells were positive for astrocyte marker GFAP as described in previous reports (Rouach et al, 2008; Giaume et al, 2010; and data not shown). For quantification and comparison, the distance of diffusion and the number of coupled cells were taken as indexes of the functional status of astrocyte GJCs. Three slices from each rat were analyzed. We also infused in the PLC two types of fluorescence dyes simultaneously: LY and rhodamine dextran (RD) (Mr 10000), which is too large to pass through the GJCs, thus serving as a tracer dye for the cells originally receiving the dye. See the Supplementary Materials and Methods for details. The results indicate that the uptake process of fluorescent molecules was not influenced by CUS (Supplementary Figure S2).

Dye staining revealed significant decreases in the distance of diffusion and the number of coupled cells in the PLC of CUS-exposed rats as compared with CTR group. Treatment with antidepressants or mifepristone significantly reversed the effect of CUS on intercellular coupling (distance, Figure $3 \mathrm{~b}$ and e; two-way ANOVA, post-hoc Newman-Keuls, for fluoxetine: stress, $F_{1,20}=65.05, P<0.0001$; drug, $\mathrm{F}_{1,20}=17.12, \quad P=0.0005 ;$ interaction, $\mathrm{F}_{1,20}=20.78, P=$ 0.0002; for duloxetine: stress, $\mathrm{F}_{1,20}=45.41, P<0.0001$; drug, $\mathrm{F}_{1,20}=33.29, \quad P<0.0001$; interaction, $\mathrm{F}_{1,20}=17.27, \quad P=$ 0.0005 ; for mifepristone: stress, $\mathrm{F}_{1,20}=49.91, P<0.0001$; drug, $F_{1,20}=6.478, P=0.0193$; interaction, $F_{1,20}=28.64$, $P<0.0001$ ) (number, Figure $3 \mathrm{c}$ and $\mathrm{f}$; two-way ANOVA, post-hoc Newman-Keuls, for fluoxetine: stress, $\mathrm{F}_{1,20}=61$, $P<0.0001$; drug, $\mathrm{F}_{1,20}=21.62, P=0.0002$; interaction, $\mathrm{F}_{1,20}=23.97, \quad P<0.0001$; for duloxetine: stress, $\mathrm{F}_{1,20}=$ 46.27, $P<0.0001$; drug, $\mathrm{F}_{1,20}=34.55, P<0.0001$; interaction, $\mathrm{F}_{1,20}=17.51, P=0.0005$; for mifepristone: stress, $\mathrm{F}_{1,20}=$ 54.58, $P<0.0001$; drug, $\mathrm{F}_{1,20}=19.15, P=0.0003$; interaction, $\left.F_{1,20}=34.25, P<0.0001\right)$, whereas antidepressants or mifepristone treatment did not affect diffusion distance and coupled number in nonstressed control rats. Our results demonstrate that CUS induces astrocyte gap junction dysfunction that can be reversed by treatment with antidepressants or mifepristone. 
a

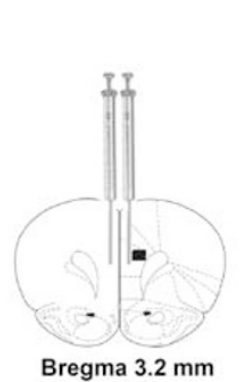

Bregma $3.2 \mathrm{~mm}$

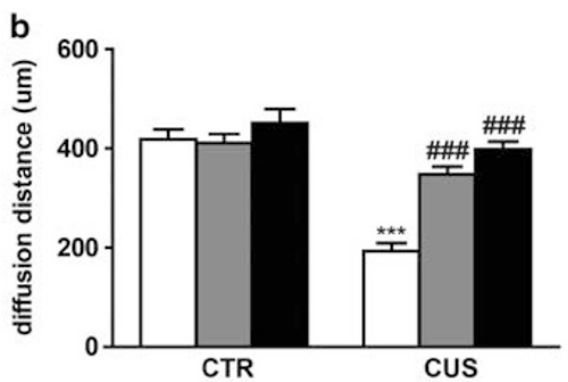

$\square$ Vehicle $\square$ Fluoxetine $\square$ Duloxetine

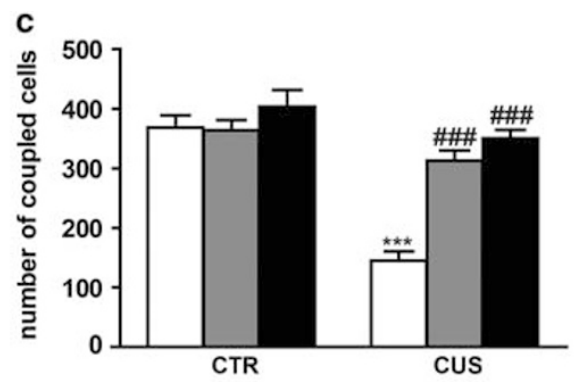

$\square$ Vehicle $\square$ Fluoxetine $\square$ Duloxetine

d
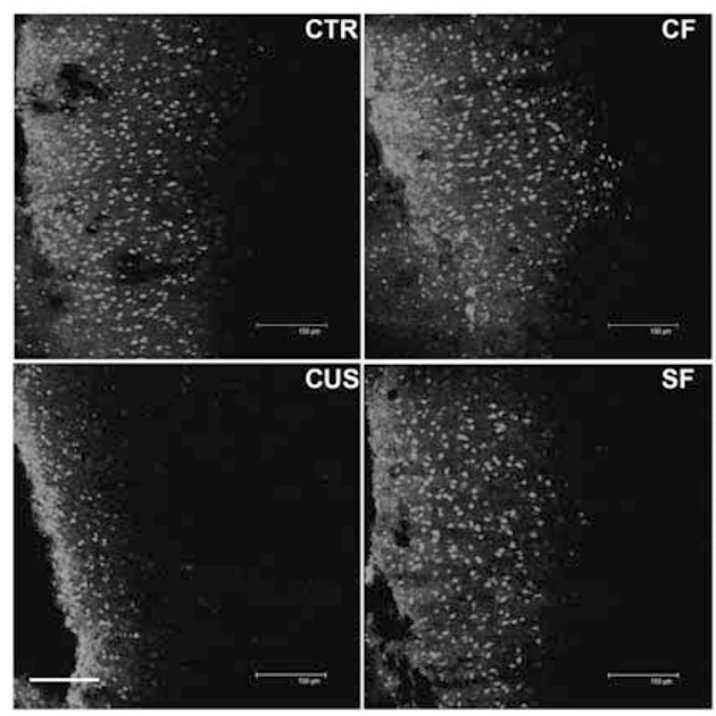
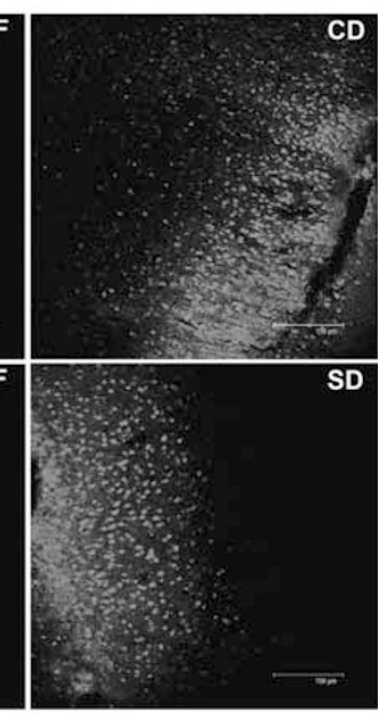
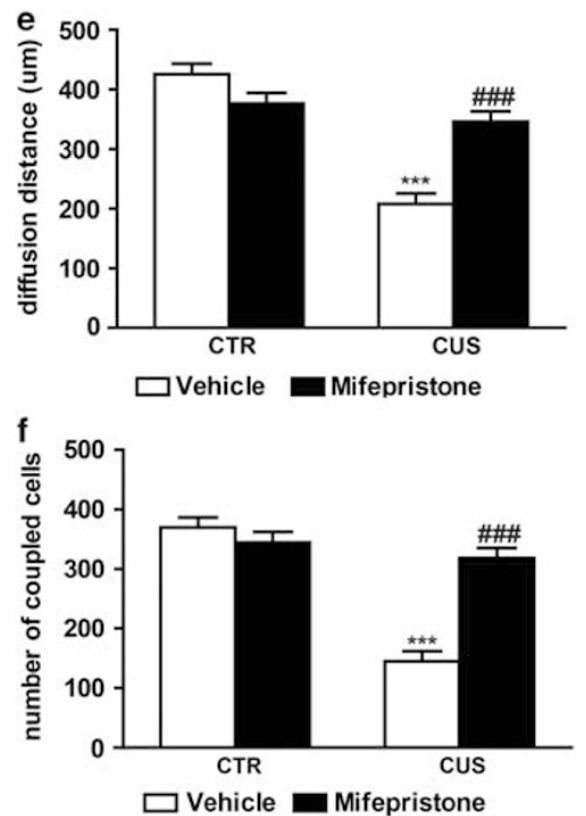

g
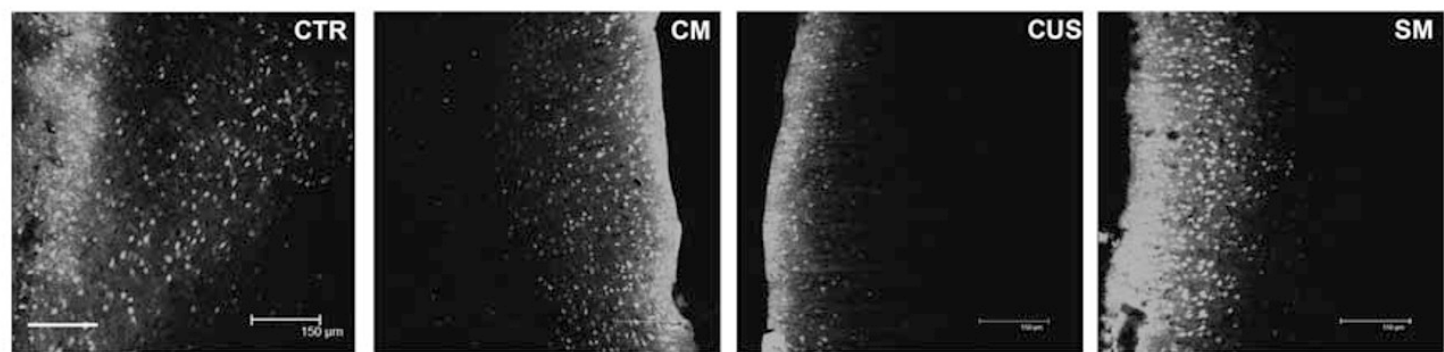

Figure 3 Antidepressants and mifepristone reversed intercellular diffusion decrease of Lucifer yellow in the prelimbic cortex (PLC) induced by chronic unpredictable stress (CUS; $n=6 /$ group). (a) The infusion illustration is shown and the distance of diffusion and the number of coupled cells in the black square are quantified (Paxinos and Watson, 1998). (b, e) The diffusion distance was significantly reduced by CUS and this effect was reversed by antidepressants or mifepristone treatment. (c, f) CUS significantly decreased the number of coupled cells and antidepressants or mifepristone significantly reversed this effect. Error bars represent SEM. ${ }^{*} * * P<0.00$ I compared with CTR and ${ }^{\# \# \# ~} P<0.00$ I compared with CUS, two-way analysis of the variance (ANOVA), Newman-Keuls post-hoc analysis. $(\mathrm{d}, \mathrm{g})$ Representative dye coupling images from animals of all groups are illustrated. Scale bar $=150 \mu \mathrm{m}$. CTR, control group; CUS, CUS group; CF, CTR + fluoxetine group; SF, CUS + fluoxetine group; CD, CTR + duloxetine group; SD, CUS + duloxetine group; CM, CTR + mifepristone group; SM, CUS + mifepristone group.

\section{Antidepressants or Mifepristone Reversed Ultrastructural Alterations of Astrocyte Gap Junction Induced by CUS}

Ultrastructural study has provided strong support for the generally accepted view that astrocytes are extensively coupled to form what are defined as 'functional syncytia' (Nagy and Rash, 2000). Detailed ultrastructural examination by electron microscopy could clearly identify the cell types linked by gap junctions. Therefore, we observed astrocyte gap junctions at the interface between two neighboring astrocytes. The structure of astrocytes and gap junctions between them in CTR animals treated with antidepressants or mifepristone and CUS animals treated with antidepressants or mifepristone was as normal as that of CTR group, whereas the cell body or process of astrocyte in CUS group was swollen and the integrity of the structure between two adjacent astrocytes was damaged (Figure $4 c$ and e). 
a

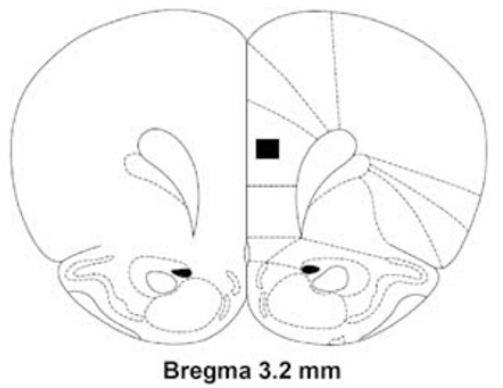

b

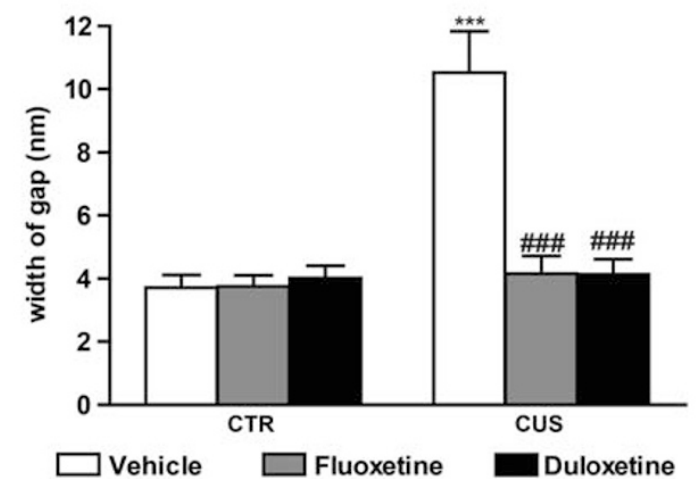

d

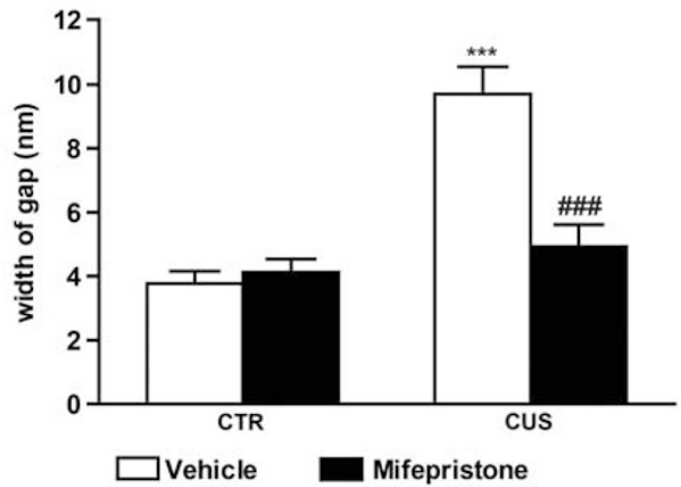

c
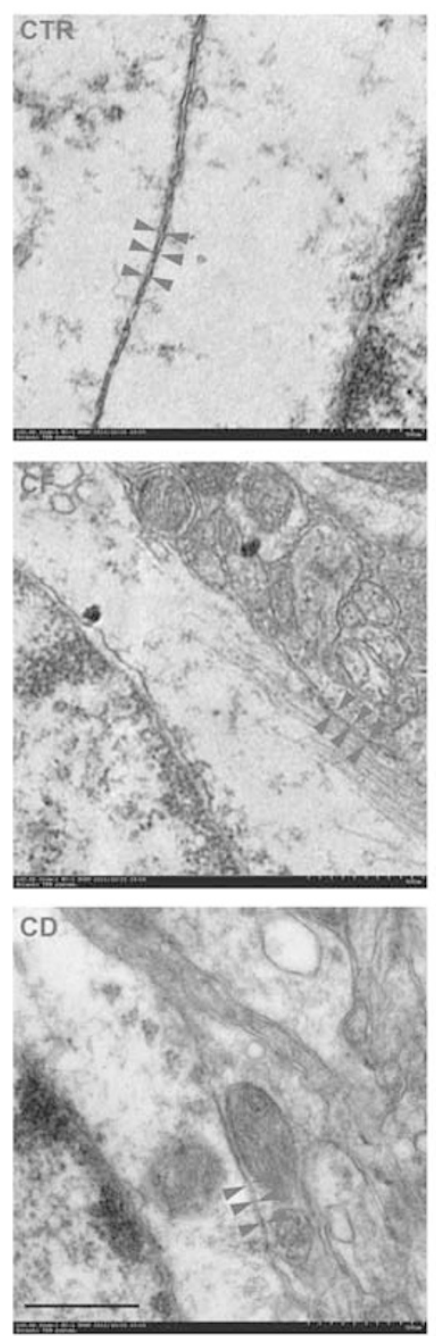

e

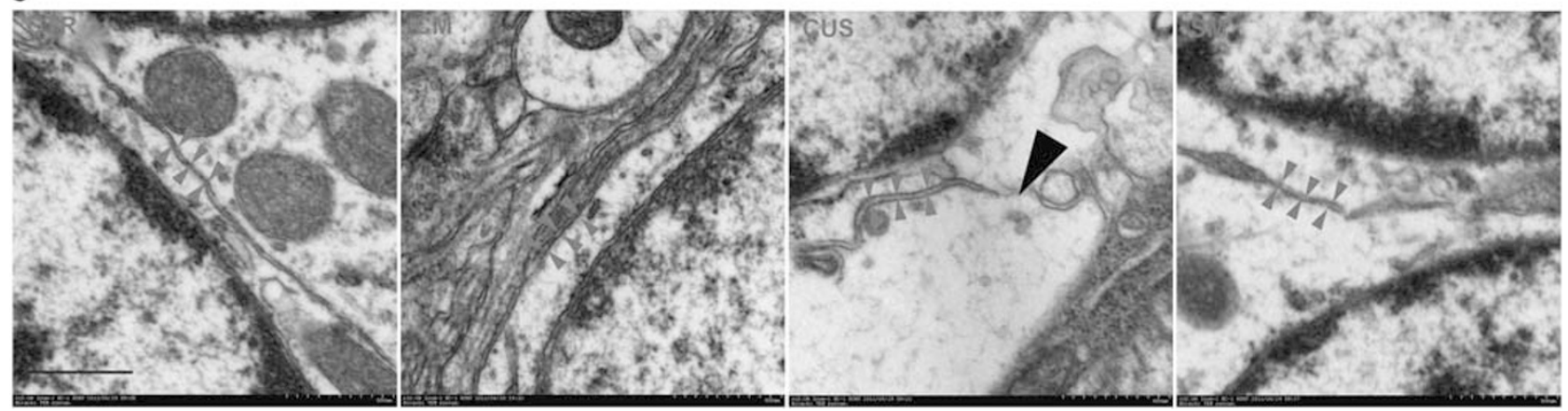

Figure 4 Antidepressants and mifepristone reversed ultrastructural alterations of astrocyte gap junction in the prelimbic cortex (PLC) induced by chronic unpredictable stress (CUS; $n=6 /$ group). (a) The sections shown in the black square were cut from the brains of all groups (Paxinos and Watson, 1998). (b, d) The width of gap was significantly enlarged by CUS and this effect was reversed by antidepressants and mifepristone treatment. Error bars represent SEM. *** $P<0.00$ I compared with CTR and ${ }^{\# \#} P<0.001$ compared with CUS, two-way analysis of the variance (ANOVA), Newman-Keuls post-hoc analysis. (c, e) Electron micrographs showing astrocytic gap junction in the PLC of each group (Magnification: $\times 100000$ ). Astrocytic gap junctions are indicated by gray arrowheads. Black arrowhead indicates the damaged structure between two adjacent astrocytes. Scale bar= $500 \mathrm{~nm}$. CTR, control group; CUS, CUS group; CF, CTR + fluoxetine group; SF, CUS + fluoxetine group; CD, CTR + duloxetine group; SD, CUS + duloxetine group; CM, CTR + mifepristone group; SM, CUS + mifepristone group.

For quantification, the width of gaps was taken as an index. The gap between two neighboring astrocytes in the PLC of CUS group was $\sim 1.5$ times wider than that of CTR group (Figure $4 \mathrm{~b}$ and $\mathrm{d}$ ). We also found that the gap width in CUS animals treated with antidepressants or mifepristone was significantly different from that in CUS animals 
(Figure 4b and d; two-way ANOVA, post-hoc NewmanKeuls, for fluoxetine: stress, $\mathrm{F}_{1,20}=22.22, P=0.0001$; drug, $\mathrm{F}_{1,20}=17.11, \quad P=0.0005 ;$ interaction, $\mathrm{F}_{1,20}=17.55, \quad P=$ 0.0005; for duloxetine: stress, $\mathrm{F}_{1,20}=20.97, P=0.0002$; drug, $\mathrm{F}_{1,20}=16.18, \quad P=0.0007$; interaction, $\mathrm{F}_{1,20}=19.57, \quad P=$ 0.0003; for mifepristone: stress, $\mathrm{F}_{1,20}=28.59, P<0.0001$; drug, $\mathrm{F}_{1,20}=12.44, P=0.0021$; interaction, $\mathrm{F}_{1,20}=16.59$, $P=0.0006$ ), whereas the gap width was similar in CTR animals treated with antidepressants or mifepristone and CTR animals (Figure $4 \mathrm{~b}$ and $\mathrm{d}$ ). The results indicate that CUS impairs the structure of astrocyte gap junction and antidepressants or mifepristone have an amelioration effect.

\section{Antidepressants or Mifepristone Reversed Decrease of Cx43 Protein and mRNA Levels Induced by CUS}

To investigate whether $\mathrm{Cx} 43$ is altered in depression, we examined the $\mathrm{Cx} 43$ protein and mRNA levels in our CUS-induced rat depression model. Cx43 was found in all brain regions, although at different levels (data not shown). Significant decreases of total $\mathrm{Cx} 43$ protein levels were found in PFC as well as in hippocampus of CUS-treated rats, whereas the $\mathrm{Cx} 43$ protein level was markedly enhanced in striatum of the stressed group. However, no significant changes were observed in other regions (Supplementary Figure S3).

Much evidence implicates the PFC as a site of pathology in depression (Rajkowska, 2000). The PFC has strong reciprocal connections with the hippocampus and other stress-related structures (Lopez et al, 1999) and may be involved in stress-mediated neurotoxicity because stress alters PFC functions and GRs (Herman et al, 1995). Hereby, we hypothesize that $\mathrm{Cx} 43$ alteration in the PFC is involved in major depression and antidepressants or mifepristone block the alteration. The results demonstrate that treatment with antidepressants or mifepristone restored the significant CUS-induced decrease of the $\mathrm{Cx} 43$ protein level in the PFC (Figure 5b and f; two-way ANOVA, post-hoc NewmanKeuls, for fluoxetine: stress, $\mathrm{F}_{1,20}=35.36, \quad P<0.0001$; drug, $\mathrm{F}_{1,20}=91.01, P<0.0001$; interaction, $\mathrm{F}_{1,20}=25.34$, $P<0.0001$; for duloxetine: stress, $\mathrm{F}_{1,20}=13.1, P=0.0017$; drug, $\mathrm{F}_{1,20}=109.7, P<0.0001$; interaction, $\mathrm{F}_{1,20}=17.16$, $P=0.0005$; for mifepristone: stress, $\mathrm{F}_{1,20}=26.42$, $P<0.0001$; drug, $\mathrm{F}_{1,20}=4.369, \quad P=0.0496$; interaction, $\left.\mathrm{F}_{1,20}=22.1, P=0.0001\right)$. In contrast to significant increases of $\mathrm{Cx} 43$ protein level directly induced by typical antidepressants, mifepristone did not have an effect alone in CTR animals (Figure $5 b$ and $\mathrm{f}$ ).

RT-PCR analysis of Cx43 in the PFC is shown in Figure 5d and $h$. Consistent with the decreased protein level of $\mathrm{Cx} 43$ in the PFC, the mRNA level in the PFC was significantly decreased in CUS-exposed rats. The decrease in mRNA level was elevated by treatment with antidepressants or mifepristone (Figure 5d and h; two-way ANOVA, post-hoc Newman-Keuls, for fluoxetine: stress, $F_{1,20}=25.48$, $P<0.0001$; drug, $\mathrm{F}_{1,20}=75.78, \quad P<0.0001$; interaction, $\mathrm{F}_{1,20}=71.01, P<0.0001$; for duloxetine: stress, $\mathrm{F}_{1,20}=60.1$, $P<0.0001$; drug, $\mathrm{F}_{1,20}=172.6, \quad P<0.0001$; interaction, $\mathrm{F}_{1,20}=48.59, \quad P<0.0001 ; \quad$ for mifepristone: stress, $\mathrm{F}_{1,20}=18.73, P=0.0003$; drug, $\mathrm{F}_{1,20}=10.4, P=0.0042$; interaction, $\left.\mathrm{F}_{1,20}=23.78, P<0.0001\right)$. Again, mifepristone did not have an effect alone in CTR animals. In contrast to the significant increase induced by duloxetine, the mRNA level of Cx43 was similar in CTR animals treated with fluoxetine and CTR animals (Figure $5 \mathrm{~d}$ and $\mathrm{h}$ ).

\section{Antidepressants or Mifepristone Reversed Decrease of the Number of Cx43-Immunoreactive Puncta Induced by CUS}

Next, Cx43 IHC was performed on the sections at the coordinates indicated of all groups (Figure 6a). Cx43immunoreactive puncta distributed in the PLC were examined and revealed a punctuate staining pattern (Figure $6 c$ and e). An obvious suppression of the number of Cx43-immunoreactive puncta occurred in the PLC in CUS-treated rats compared with control rats (Figure 6b and $\mathrm{d}$ ). Treatment with antidepressants or mifepristone restored the significant decrease of the $\mathrm{Cx} 43$ puncta number (Figure 6b and d; two-way ANOVA, post-hoc NewmanKeuls, for fluoxetine: stress, $\mathrm{F}_{1,20}=105.4, \quad P<0.0001$; drug, $F_{1,20}=31.89, P<0.0001$; interaction, $F_{1,20}=17.17$, $P=0.0005$; for duloxetine: stress, $F_{1,20}=57.3, P<0.0001$; drug, $\quad \mathrm{F}_{1,20}=55.09, \quad P<0.0001$; interaction, $\mathrm{F}_{1,20}=$ $10.9, P=0.0036$; for mifepristone: stress, $\mathrm{F}_{1,20}=43.77$, $P<0.0001$; drug, $\mathrm{F}_{1,20}=11.82, \quad P=0.0026$; interaction, $\left.\mathrm{F}_{1,20}=27.55, P<0.0001\right)$. Mifepristone had no effect on the density of $\mathrm{Cx} 43$-immunoreactive puncta in nonstressed animals, whereas the density of puncta was significantly increased in CTR animals treated with duloxetine and revealed an increasing tendency in CTR animals treated with fluoxetine compared with CTR animals (Figure 6b and $\mathrm{d}$ ). This alteration was homogeneous and was observed throughout the anterior-posterior extent of the PLC (Figure 6a).

In summary, both the functional and structural alterations of astrocytic gap junction and the alterations of $\mathrm{Cx} 43$ were observed in CUS-treated animals and restored after treatment with antidepressants or mifepristone.

\section{Gap Junction Blockade in the PFC Induces Depressive-Like Behaviors}

To determine whether gap junction dysfunction in the PFC contributes to the expression of depressivelike behaviors, we analyzed the behavioral effects of CBX infusions in the PLC (Figure 7a) on SPT and NSFT. $\mathrm{CBX}$ is an extensively used gap junction blocker that does not have significant interference with synaptic function (Ross et al, 2000; Gajda et al, 2003), nor does it significantly alter intrinsic neuronal characteristics (Frantseva et al, 2002). CBX was previously found to downregulate the expression of $\mathrm{Cx} 43$ in vitro in $24 \mathrm{~h}$ (Herrero-Gonzalez et al, 2009) and to have behavioral and cellular effects in vivo in 1 to 3 days (Miguel-Hidalgo et al, 2009; Xie et al, 2011). Furthermore, it was used to inhibit gap junctional coupling in a wide range of doses in vivo, from $150 \mu \mathrm{M} \times$ $2 \mu \mathrm{l}$ to $150 \mathrm{mM} \times 4 \mu \mathrm{l}$ (Nilsen et al, 2006; Kokarovtseva et al, 2009). Therefore, we infused three doses of CBX $(1,10$, and $100 \mathrm{mM} \times 1 \mu \mathrm{l}$ ) on day 2, and monitored sucrose preference $24 \mathrm{~h}$ later. Animals treated with CBX bilaterally showed significant decreases in the ratio of the volume of sucrose $v s$ total volume of sucrose and water consumed 
a
Cx43 CTR CF CD CUS SF SD

$C \times 43$

$\beta$-actin
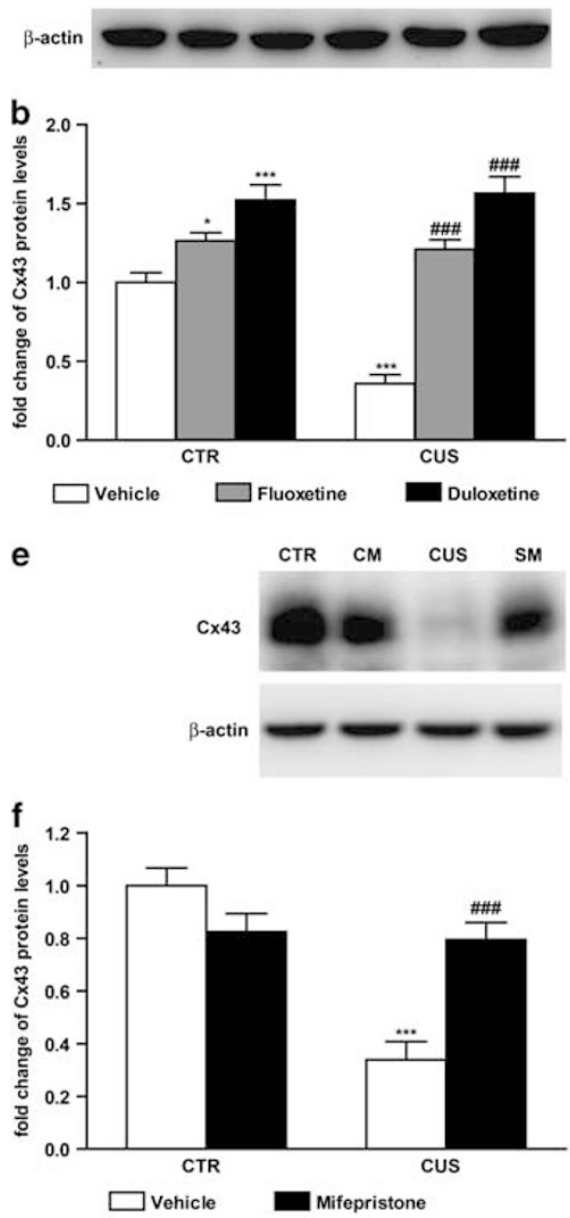
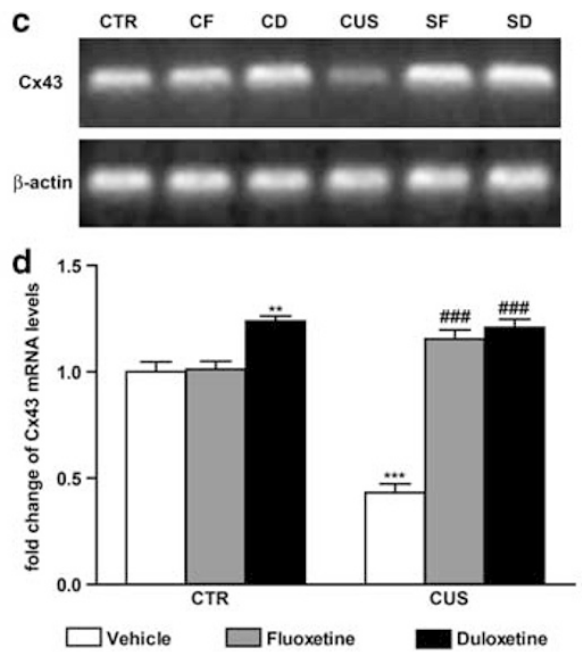

g

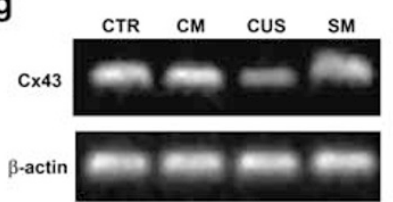

h

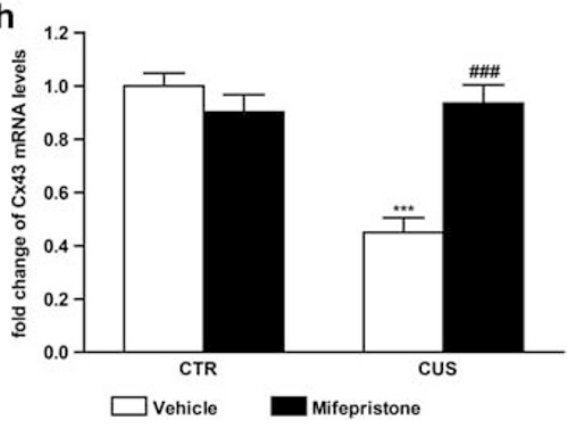

Figure 5 Antidepressants and mifepristone reversed decreases of connexin 43 (Cx43) protein and mRNA levels in the prefrontal cortex (PFC) induced by chronic unpredictable stress (CUS; $n=6 /$ group). The protein levels of $\mathrm{C} \times 43$ were determined by western blot. (a, e) Representative western blot images of $\mathrm{C} \times 43$ and $\beta$-actin of each group are shown, and $(\mathrm{b}, \mathrm{f})$ results were quantified and are the mean $\pm \mathrm{SEM}$, taking the ratio of the gray level of $\mathrm{C} \times 43$ band to the $\beta$-actin band of control group as unity. CUS significantly decreased the protein levels of CX43 and antidepressants or mifepristone significantly reversed this effect. The mRNA levels of $\mathrm{C} \times 43$ were determined by RT-PCR. (c, g) Representative gel patterns of $C \times 43$ and $\beta$-actin PCR product bands of each group are shown. $(d, h)$ Relative amount (mean \pm SEM) of Cx43 mRNA is shown, as percent of CTR. CUS significantly decreased the mRNA levels of Cx43 and antidepressants and mifepristone significantly reversed this effect. $* P<0.05$, $* * P<0.01$, $* * * P<0.001$ compared with $C T R$ and $\# \# \# P<0.001$ compared with CUS, two-way analysis of the variance (ANOVA), Newman-Keuls post-hoc analysis. RT-PCR, reverse transcriptase-PCR; CTR, control group; CUS, CUS group; CF, CTR + fluoxetine group; SF, CUS + fluoxetine group; CD, CTR + duloxetine group; SD, CUS + duloxetine group; CM, CTR + mifepristone group; SM, CUS + mifepristone group.

compared with PBS-infused animals (Figure 7b; one-way ANOVA, $\left.\mathrm{F}_{3,20}=10.95, P=0.0002\right)$. The effects of 1,10 , and $100 \mathrm{mM} \times 1 \mu \mathrm{l}$ CBX infusions on SPT were dose dependent, decreases of $47.8 \%, 63.3 \%$, and $70.5 \%$, respectively. However, total fluid consumption did not differ during the 1 -h test, nor did water consumption monitored for the next $24 \mathrm{~h}$ (data not shown).

After the SPT, food was removed, and $24 \mathrm{~h}$ later, the effects of CBX on NSFT were determined. We found significant increases in latency to feed in the 1,10 , and $100 \mathrm{mM} \times 1 \mu \mathrm{l}$ CBX-infused animals, $71.5 \%, 102.3 \%$, and $122.9 \%$, respectively (Figure $7 \mathrm{c}$; one-way ANOVA, $\mathrm{F}_{3,20}=5.736, P=0.0053$ ). There was no difference in home cage food consumption (Supplementary Figure S4).
Figure 7d illustrates the effects of $\mathrm{Cx} 43$ mimetic peptides Gap27 and Gap26 on SPT on day 3. Gap26 and Gap27 were designed corresponding to sequences in the first and second extracellular loops, respectively, of $\mathrm{Cx} 43$ (Evans and Leybaert, 2007), and therefore they have greater selectivity to inhibit gap junctions composed of $\mathrm{Cx} 43$, mostly astrocytic gap junction. Animals treated with Gap27 and Gap26 showed significant decreases in the ratio of sucrose solution vs total liquid consumed compared with PBSinfused animals, whereas scrambled control peptide had no effect (Figure 7d; one-way ANOVA, $\mathrm{F}_{3,20}=6.494$, $P=0.003$ ). Animals in which the track of the infusion needle missed the PLC were excluded from the statistical analysis (Supplementary Figure S5). 
a

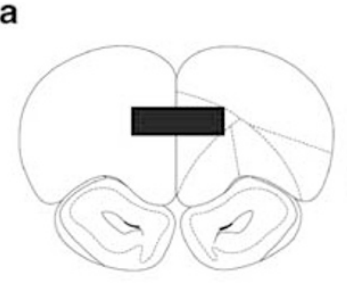

From $4.2 \mathrm{~mm}$

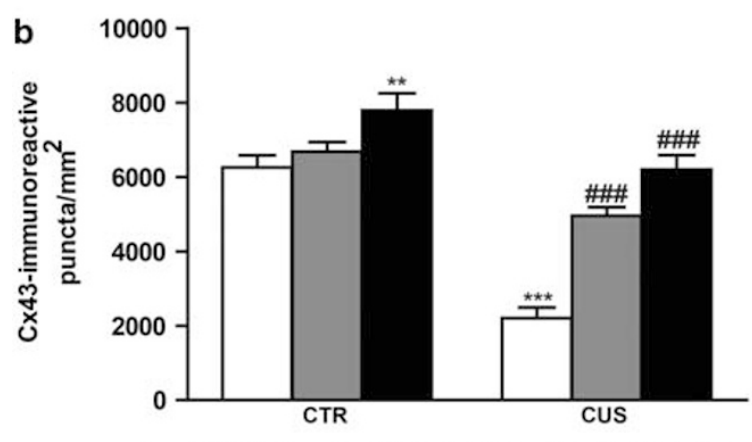

$\square$ Vehicle $\square$ Fluoxetine
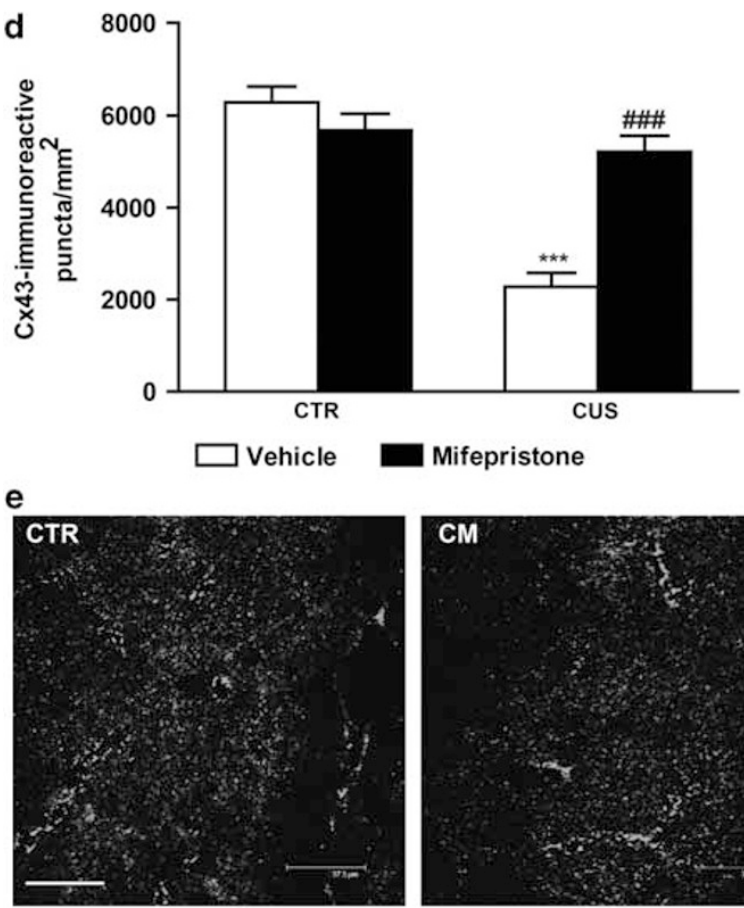

\section{CM}

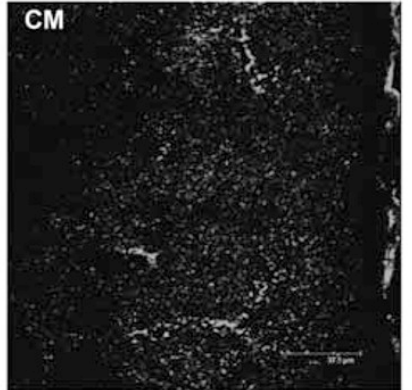

C
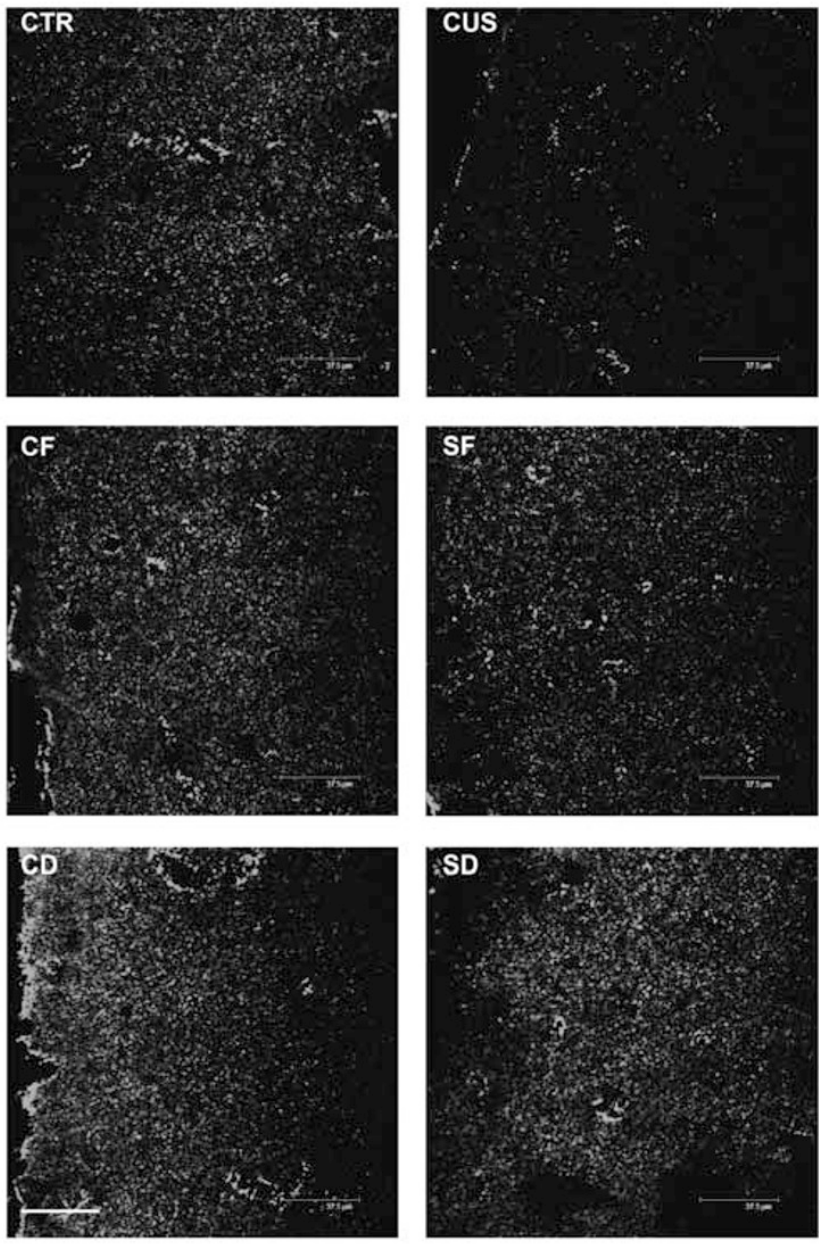

Figure 6 Antidepressants and mifepristone reversed decrease of the number of connexin 43 (Cx43)-immunoreactive puncta in the prelimbic cortex (PLC) induced by chronic unpredictable stress (CUS; $n=6$ /group). Numbers of Cx43-immunoreactive puncta were quantified throughout the PLC as shown in (a) (black squares) (Paxinos and Watson, 1998). (b, d) The number of Cx43-immunoreactive puncta/mm² was significantly decreased by CUS and this effect was reversed by antidepressants or mifepristone treatment. Error bars represent SEM. $* * P<0.0$ I, **** $P<0.00$ I compared with $C T R$ and $\# \# \# P<0.001$ compared with CUS, two-way analysis of the variance (ANOVA), Newman-Keuls post-hoc analysis. (c, e) Representative Cx43-stained sections from animals of all groups are illustrated at $\sim 3.2 \mathrm{~mm}$ from bregma. Scale bar $=37.5 \mu \mathrm{m}$. CTR, control group; CUS, CUS group; CF, CTR + fluoxetine group; SF, CUS + fluoxetine group; CD, CTR + duloxetine group; SD, CUS + duloxetine group; CM, CTR + mifepristone group; SM, CUS + mifepristone group.

CBX Did Not Affect the Density of GFAP-Positive Cells in the PLC

Infusion of PBS in the control experiments for CBX resulted in small lesions in the PLC when observed 3 days after the surgery. In these sites infused with vehicle, neurons appeared intact around the needle track and GFAPimmunostained astrocytes were slightly enlarged as a result of reactive gliosis to needle placement without other obvious damages (data not shown). We quantified the 
a
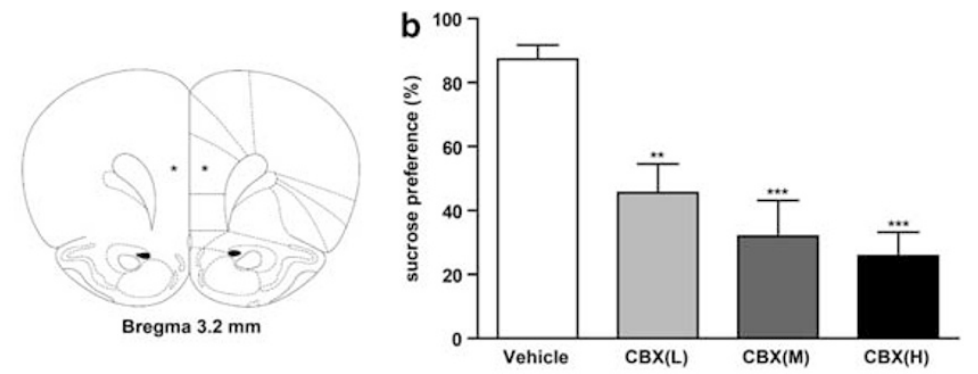
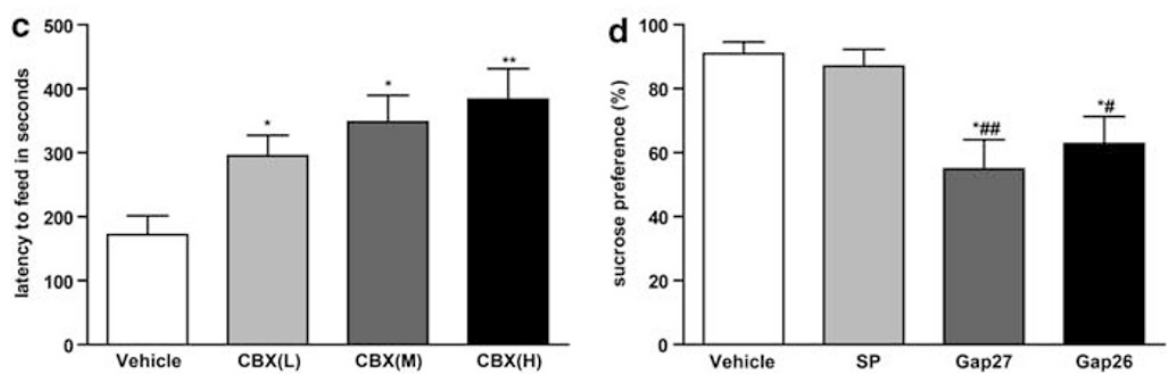

Figure 7 Gap junction blockade in the prefrontal cortex (PFC) induces depressive-like behaviors. (a) The infusion site (coordinates ( $m m$ ): anteroposterior + 3.2, dorsolateral -0.5, depth -4 from Bregma) is marked by an asterisk (*) (Paxinos and Watson, 1998). (b, c) Effects of carbenoxolone (CBX) infusions on sucrose preference test (SPT) and novelty suppressed feeding test (NSFT; $n=6 /$ group). Animals received infusions of phosphate-buffered saline (PBS, Vehicle), I mM of CBX (low-dose CBX, CBX(L)), I 0 mM of CBX (medium-dose CBX, CBX(M)), or 100 mM of CBX (high-dose CBX, CBX(H)) and were consecutively tested in SPT (day 3) and NSFT (day 4). Results are expressed as mean \pm SEM ratio of sucrose solution vs total liquid consumed (b) and latency to feed in seconds (c). $* P<0.05$, $* * P<0.01$, **** $P<0.00$ I compared with Vehicle. (d) Effects of connexin 43 (Cx43) mimetic peptide Gap27 and Gap26 infusions on SPT ( $n=6 /$ group). PBS (Vehicle), scrambled peptide (SP), Gap27, or Gap26 were bilaterally infused and sucrose preference was measured. $* P<0.05$ compared with SP and ${ }^{\#} P<0.05,{ }^{\# \#} P<0.01$ compared with Vehicle.

a

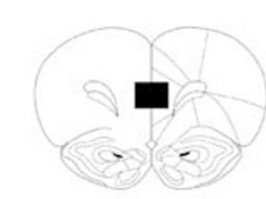

From $3.7 \mathrm{~mm}$
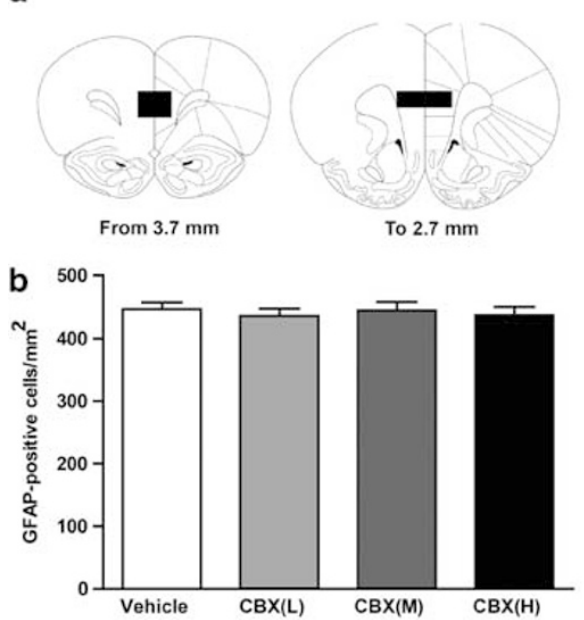

C
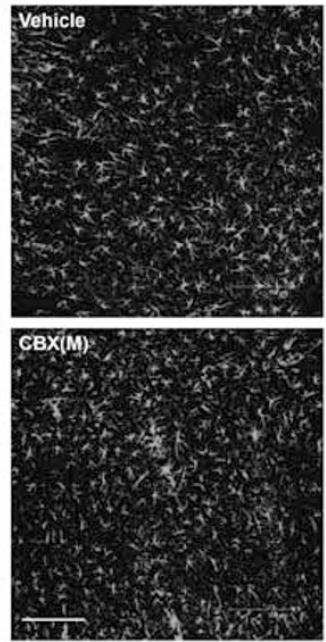
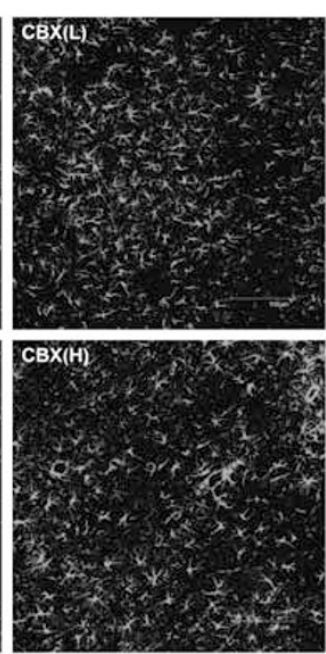

Figure 8 Influence of carbenoxolone (CBX) infusions in the prefrontal cortex (PFC) on density of glial fibrillary acidic protein (GFAP)-positive cells $(n=6 /$ group). (a) Anterior-to-posterior stereotaxic coordinates of representative coronal brain slices are shown (Paxinos and Watson, 1998). Numbers of GFAPpositive cells were quantified in the prelimbic cortex (PLC) as shown in (a) (black squares). (b) Results are presented as mean \pm SEM, and there was no significant difference in all groups in the number of GFAP-positive cells $/ \mathrm{mm}^{2}$ (one-way analysis of the variance $(A N O V A), F_{3,20}=0.1988, P=0.896$ ). (c) Representative GFAP immunofluorescence-stained sections from each group are shown at $\sim 3.7 \mathrm{~mm}$ from bregma, away from the infusion site. Scale bar $=150 \mu \mathrm{m}$. Vehicle, PBS-infused group; $C B X(L)$, low-dose (I mM) CBX-infused group; CBX(M), medium-dose (I 0 mM) CBX-infused group; CBX(H), high-dose ( $100 \mathrm{mM})$ CBX-infused group.

effects of CBX infusions on the density of GFAP-positive cells in the PLC of animals from experiment 3. After infusions of different doses of CBX, GFAP-immunoreactive astrocytes appeared very similar to those in vehicle-infused animals (Figure 8b; one-way ANOVA, $\mathrm{F}_{3,20}=0.1988$,
$P=0.896$ ), also without apparent damage to surrounding neurons. More precisely, no significant differences in the number of GFAP-positive cells $/ \mathrm{mm}^{2}$ were observed at levels where the needle tracks were visible, at levels from 3.4 to $3.0 \mathrm{~mm}$ from bregma, or at anterior-posterior levels away 


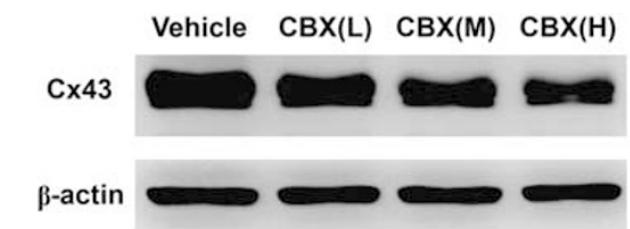

b

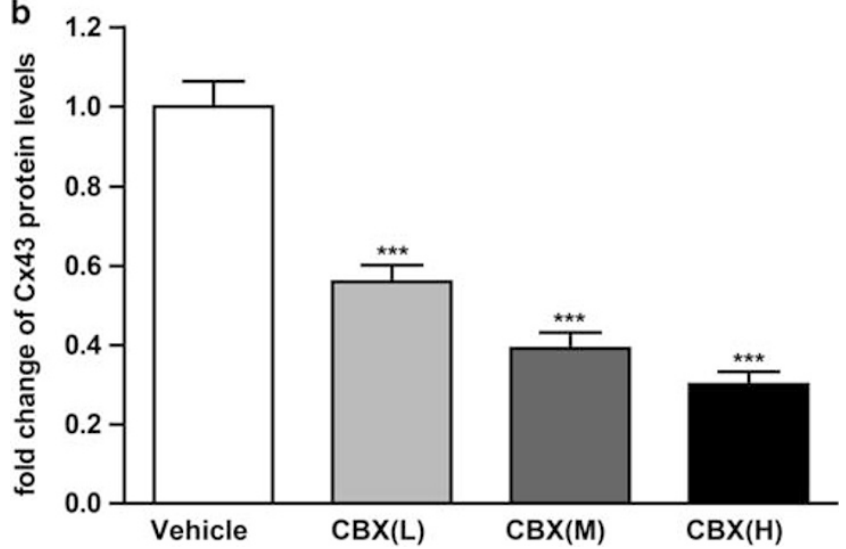

Figure 9 Effects of carbenoxolone (CBX) infusions in the prefrontal cortex (PFC) on the protein level of connexin 43 (Cx43;n=6/group). The protein levels of $\mathrm{C} \times 43$ in the PFC of each group were determined. (a) Representative western blot images of $\mathrm{C} \times 43$ and $\beta$-actin are shown, and (b) results were quantified and are the mean \pm SEM, taking the ratio of the gray level of $\mathrm{C} \times 43$ band to the $\beta$-actin band of Vehicle as unity. **** $P<0.001$ compared with Vehicle. Vehicle, PBS-infused group; $C B X(L)$, low-dose (I mM) CBX-infused group; $C B X(M)$, medium-dose ( $10 \mathrm{mM})$ $\mathrm{CBX}$-infused group; $\mathrm{CBX}(\mathrm{H})$, high-dose ( $100 \mathrm{mM}) \mathrm{CBX}$-infused group.

from the needle track corresponding to levels 3.7 to $3.4 \mathrm{~mm}$ and 3.0 to $2.7 \mathrm{~mm}$ from bregma, respectively.

CBX did not induce a noticeable glial loss in the PLC, determined by analysis of GFAP-positive cells, although there were signs of gap junction impairment, including the obvious decrease of Cx43 protein level (Figure 9b; one-way ANOVA, $\left.\mathrm{F}_{3,20}=46.42, P<0.0001\right)$. Consistent with the behavioral effects of multiple doses of CBX, the effects of CBX on $\mathrm{Cx} 43$ protein level were also dose dependent.

\section{DISCUSSION}

This study provides the first experimental evidence of cortical astrocyte gap junction dysfunction in an animal model of depression. The results demonstrate that animals exposed to CUS exhibit behavioral deficits in tests measuring anhedonia and anxiety, as well as functional and ultrastructural deficits of astrocytic gap junction and reductions in $\mathrm{Cx} 43$ expression and $\mathrm{Cx} 43$ puncta density in the PFC. The behavioral and cellular alterations induced by CUS were reversed or blocked both by chronic treatment with the typical antidepressant fluoxetine or duloxetine or by subchronic treatment with the GR antagonist mifepristone. First, the beneficial effects of mifepristone on CUSinduced anhedonia and anxiety clearly define mifepristone as an antidepressant treatment in this model. Second, the cellular mechanism of their antidepressant action may involve the amelioration of gap junction dysfunction. Third, the cellular changes induced by CUS may be related to GR activation. The results also demonstrate that pharmacological blockade of gap junction in the PFC produces depressive-like behaviors without glial ablation. More precisely, we demonstrate that both CBX and Cx43 mimetic peptide-induced gap junction uncoupling in the PFC are sufficient to induce behavioral deficits similar to those induced by CUS. Together, our data support the hypothesis that gap junction dysfunction in the PFC may contribute to the expression of the depressive-like symptoms.

The CUS paradigm is an animal model of depression with high predictive, face, and construct validity (Willner, 2005), and is thought to simulate stressful life events that promote the development of human depression and produce behavioral and neuroendocrine outcomes similar to that observed in depressed patients (Willner et al, 1987, 1997). We confirmed that this stress sequence reduces sucrose preference, assessing the hedonic state of stressed animals (Willner et al, 1992), and we show that the same procedure induces anxiety, demonstrated by increased latency to feed in NSFT. The behavioral alterations induced by CUS were reversed by chronic treatment with typical antidepressants fluoxetine and duloxetine as expected, and mifepristone treatment significantly increased sucrose preference, consistent with a previous study (Wu et al, 2007). In addition, we extended the behavioral characterization and demonstrate that mifepristone has an ameliorative effect on anxiety-like behaviors in the NSFT.

Growing evidence in clinical and preclinical studies has implicated glial anomalies in the pathophysiology of MDD (Rajkowska and Miguel-Hidalgo, 2007). Reduced number and altered morphology of glial cells in the PFC were described in many studies (Ongur et al, 1998; Cotter et al, 2001, 2002; Banasr and Duman, 2008), whereas impaired cortical glial function was rarely reported (Banasr et al, 2010). In this study, we demonstrate that CUS impairs the intercellular coupling of GJC-permeable dye LY in the PLC. In line with the previous reports (Giaume et al, 2010), astrocytes are the main cell type responsible for diffusion of the fluorescent molecule in the brain; the lower dye labeling in the PLC indicates that astrocytic gap junction is impaired in animals subjected to CUS. EM studies show that the ultrastructure of astrocyte gap junction in the PFC of CUS animals is abnormal, providing another strong support for the view that CUS exposure has deleterious effects on astrocytic gap junction in the PFC.

In the adult brain, $\mathrm{Cx} 43$ and $\mathrm{Cx} 30$ are the main $\mathrm{Cxs}$ in astrocytes (Giaume et al, 1991; Kunzelmann et al, 1999). They were identified as the molecular constituents of GJCs. Less data are available on the bioactive molecules that can permeate through $\mathrm{Cx} 30 \mathrm{GJCs}$, whereas $\mathrm{Cx} 43$ channels are selective for several endogenous molecules, including second messengers (cyclic AMP, inositol-1,4,5-trisphosphate (Ins $\mathrm{P}_{3}$ ), and $\left.\mathrm{Ca}^{2+}\right)$, amino acids (glutamate, aspartate, and taurine), nucleotides (ADP, ATP, CTP, and NAD), energy metabolites (glucose, glucose-6-phosphate, and lactate), small peptides (glutathione), and RNA (24-mer) (Harris, 2007; Giaume et al, 2010). Expression-function correlations for $\mathrm{Cx} 43$ have been provided in several models. For example, an increase in $\mathrm{Cx} 43$ expression and gap junctional communication was described in epileptic situations or in a model of Alzheimer's disease (Samoilova et al, 2003; Peters et al, 2009). Therefore, we investigated 
Cx43 alterations to further evaluate the function of astrocyte gap junction. The results demonstrate that CUS causes reductions of $\mathrm{Cx} 43$ protein and mRNA levels, as well as reductions in the density of $\mathrm{Cx} 43$-immunoreactive puncta in the PFC. Alterations of $\mathrm{Cx} 43$ are consistent with the results obtained in our dye transfer analysis.

At the cellular level, chronic treatment with both SSRI fluoxetine and SNRI duloxetine increased $\mathrm{Cx} 43$ expression in the PFC. Although we did not find significant alterations in expression of $\mathrm{Cx} 43 \mathrm{mRNA}$ and $\mathrm{Cx} 43$ puncta density in CTR animals treated with fluoxetine, we detected the significant increase of $\mathrm{Cx} 43$ protein level, consistent with a previous report (Fatemi et al, 2008). The increased Cx43 expression may provide a mechanism contributing to the increased dye diffusion that is observed following antidepressants treatment. Chronic antidepressants treatment also prevented or reversed the CUS-induced dysfunction of astrocyte gap junction in the PFC as the reduction in dye diffusion, the abnormal ultrastructure, and decrease in Cx43 expression found after chronic stress were reversed by fluoxetine and duloxetine. These results support the hypothesis that astrocyte gap junction dysfunction in the PFC contributes to the expression of the symptoms of MDD.

Patients with psychotic major depression are characterized by abnormalities in the HPA axis activity (Belanoff et al, 2002). The rat model of depression induced by CUS also shows signs of increased activity in the HPA axis, such as corticosterone hypersecretion (Banasr et al, 2007), which may support the validity of the CUS-induced rat model of psychotic depression. GR antagonist mifepristone is now in Phase III clinical trials for psychotic major depression (Corcept Therapeutics) and several investigations suggest that mifepristone leads to the rapid amelioration of psychotic depression (Belanoff et al, 2001, 2002; Simpson et al, 2005; Flores et al, 2006; DeBattista et al, 2006). In CUS animals, the behavioral alterations induced by CUS were reversed by repeated mifepristone treatment. The beneficial effects of mifepristone on CUS-induced anhedonia and anxiety clearly define mifepristone as an antidepressant and further validate the findings of several open-label clinical studies demonstrating the efficacy of mifepristone's in the treatment of psychotic depression. In addition, a recent work demonstrated that nongenomic glucocorticoid receptor action regulates gap junction intercellular communication in NPCs (Samarasinghe et al, 2011). Hereby, we hypothesize that mifepristone blocks the dysregulation of gap junction in the PFC as a GR antagonist. We found that the functional and ultrastructural deficits of astrocytic gap junction and reductions in $\mathrm{Cx} 43$ expression and $\mathrm{Cx} 43$ puncta density in the PFC induced by 28 days of CUS were normalized after 7 days of mifepristone treatment. The results also suggest that astrocyte gap junction dysfunction induced by CUS in the PFC depends on GR activation, in line with the proposal that stress and an excess of glucocorticoids could lead initially to the pathology of glial cells, and consequently to the pathology of neurons as depressive illness progresses (Rajkowska, 2000; Rajkowska and Miguel-Hidalgo, 2007). We also found a specific interaction between chronic stress and mifepristone treatment, indicating that the treatment interferes directly with the consequences of chronic stress exposure: mifepristone treatment alone is ineffective in handled controls, but prevents or reverses the cellular alteration seen after chronic stress. The specificity for a high-stress environment may be due to the ability of mifepristone to directly bind and block GRs that are normally only fully occupied when circulating corticosterone levels are high. At low corticosteroid levels, GRs are not substantially occupied and blockade by mifepristone would then be less effective. Together, the results suggest that dysregulation of the function of astrocyte gap junction in the PFC is associated with stress and depression and may contribute to the expression of the depressive-like symptoms.

Given the role of gap junctions in the buffering of ions, long-range signaling, and exchange of small molecules in astroglial networks (Theis et al, 2005; Scemes and Giaume, 2006; Wallraff et al, 2006; Cruz et al, 2007), astroglial GJCs may play an important role in allowing information processing and integration from a large number of neurons, in providing metabolites to remote sites during high neuronal demand, and in buffering ion or neurotransmitter concentrations (Giaume et al, 2010). In particular, owing to the proximity of astrocyte gap junctions and neuronal synapses, the concept of the tripartite synapse was initiated (Araque et al, 1999; Perea et al, 2009) and astrocytic networks, thanks to their permeability to $\mathrm{Ca}^{2+}$ and the potential gliotransmitters such as glutamate, could coordinate the activity of local groups of synapses (Giaume et al, 2010). Hereby, it is plausible that impaired GJIC in astrocytes may result in altered neurotransmission in the PFC, and contribute to malfunction in circuits involved in emotion-related subcortical centers, heavily connected with the PFC and directly involved in the pathophysiology of MDD.

Current theories propose that reductions of cortical or hippocampal glia contribute to neuronal dysfunction responsible for the expression of the depressive-like symptoms (Rajkowska and Miguel-Hidalgo, 2007; Banasr and Duman, 2008). We also detected remarkable reductions of GFAP protein and mRNA levels, as well as astrocyte loss when visualized by GFAP immunoreactivity in the PFC of stressed rats, in accordance with the previous reports (Banasr and Duman, 2008; Banasr et al, 2010). Altered number and morphology of glial cells may be the cause or consequence of glial dysfunction in stressed rats. The central question is whether the impairment of gap junction plays a part in causing the development of depression or whether it is merely an associated phenomenon to glial loss. Therefore, we investigated the effects of gap junction inhibition in the PFC on depressive-like behaviors using a gap junction blocker $\mathrm{CBX}$. The results demonstrate that CBX infusions do not decrease the density of astrocytes in the PFC, nor provoke a reduction of the length and complexity of the processes of GFAP-positive cells, although there were signs of gap junction impairment, including the obvious decreases of Cx43 protein level. The CBX-induced gap junction blockade is associated with reduced sucrose preference and increased latency to feed, phenotypes that usually take weeks to appear when animals are subjected to stress (Willner et al, 1987; Santarelli et al, 2001). In accordance with the dose-dependent alterations of $\mathrm{Cx} 43$ protein level, the effects of CBX on SPT and NSFT are also dose dependent. CBX is not a completely specific gap junctional uncoupler, as it is known to enhance the effects 
of endogenous glucocorticoid hormones by inhibiting 11 $\beta$-hydroxysteroid dehydrogenase (Bujalska et al, 1997). However, this effect can be negligible in this study, because glucocorticoid can only induce these depressive-like behaviors in several weeks, whereas the effects of CBX were detected just 24 or $48 \mathrm{~h}$ later. We also infused $\mathrm{Cx} 43$ mimetic peptides Gap27 and Gap26, two astrocyte-specific gap junction blockers in the PFC, and monitored sucrose preference known to assess anhedonia, a core symptom of depression (Willner et al, 1992). Our observations support the notion that gap junction dysfunction in the rat PFC contributes to the expression of depressive-like symptoms.

This study is a first step toward understanding the role of cortical gap junction dysfunction in the pathophysiology of depression. Our study could help clarify the pathogenesis of depression and thus benefit antidepressant drug development. However, it also raises methodological concerns that need to be mentioned, and some tasks for future investigations are listed below. First, the gap junction blockers that we used in the study have obvious limitations: they block hemichannels as well as GJCs and do not allow their respective roles to be distinguished. Cx-knockout mice or molecular tools may be alternative strategies (Giaume and Theis, 2010). Second, better methods of examining the functions of GJCs should be developed: observing the trafficking of bioactive molecules not inactive tracers through GJCs represents another important challenge; $\mathrm{Cx}$-immunogold electron microscopic localization might be more convincing; $\mathrm{Cx} 30$ is also a major gap junction protein in astrocytes, and hence it will be necessary to examine changes in $\mathrm{Cx} 30$. Third, we found that $\mathrm{Cx} 43$ protein level changed differently in several brain regions. This site-specific susceptibility of $\mathrm{Cx} 43$ induction by CUS could be of great importance, as the great heterogeneity of astrocytes including different densities and characteristics among brain sites is a key to understanding MDD pathology (Gosselin et al, 2009). The approaches above will ultimately lead to a more complete understanding of the role of glial gap junctions in depression.

\section{ACKNOWLEDGEMENTS}

This work was supported by National Natural Science Foundation of China Grants (no. 30973887, no. 81073078, and key program no. U832008), National Key Sci-Tech Major Special Item (no. 2012ZX09301002-004), and Graduate Innovation Fund 2010 of Peking Union Medical College.

\section{DISCLOSURE}

The authors declare no conflict of interest.

\section{REFERENCES}

Araque A, Parpura V, Sanzgiri RP, Haydon PG (1999). Tripartite synapses: glia, the unacknowledged partner. Trends Neurosci 22: 208-215.

Ayensu WK, Pucilowski O, Mason GA, Overstreet DH, Rezvani AH, Janowsky DS (1995). Effects of chronic mild stress on serum complement activity, saccharin preference, and corticosterone levels in Flinders lines of rats. Physiol Behav 57: 165-169.
Banasr M, Chowdhury GM, Terwilliger R, Newton SS, Duman RS, Behar KL et al (2010). Glial pathology in an animal model of depression: reversal of stress-induced cellular, metabolic and behavioral deficits by the glutamate-modulating drug riluzole. Mol Psychiatry 15: 501-511.

Banasr M, Duman RS (2008). Glial loss in the prefrontal cortex is sufficient to induce depressive-like behaviors. Biol Psychiatry 64: 863-870.

Banasr M, Valentine GW, Li XY, Gourley SL, Taylor JR, Duman RS (2007). Chronic unpredictable stress decreases cell proliferation in the cerebral cortex of the adult rat. Biol Psychiatry 62: 496-504.

Belanoff JK, Flores BH, Kalezhan M, Sund B, Schatzberg AF (2001). Rapid reversal of psychotic depression using mifepristone. J Clin Psychopharmacol 21: 516-521.

Belanoff JK, Rothschild AJ, Cassidy F, DeBattista C, Baulieu EE, Schold C et al (2002). An open label trial of C-1073 (mifepristone) for psychotic major depression. Biol Psychiatry 52: 386-392.

Bujalska I, Shimojo M, Howie A, Stewart PM (1997). Human 11 beta-hydroxysteroid dehydrogenase: studies on the stably transfected isoforms and localization of the type 2 isozyme within renal tissue. Steroids 62: 77-82.

Cotter D, Mackay D, Chana G, Beasley C, Landau S, Everall IP (2002). Reduced neuronal size and glial cell density in area 9 of the dorsolateral prefrontal cortex in subjects with major depressive disorder. Cereb Cortex 12: 386-394.

Cotter D, Mackay D, Landau S, Kerwin R, Everall I (2001). Reduced glial cell density and neuronal size in the anterior cingulate cortex in major depressive disorder. Arch Gen Psychiatry 58: 545-553.

Cruz NF, Ball KK, Dienel GA (2007). Functional imaging of focal brain activation in conscious rats: impact of [(14)C]glucose metabolite spreading and release. J Neurosci Res 85: 3254-3266.

Czeh B, Simon M, Schmelting B, Hiemke C, Fuchs E (2006). Astroglial plasticity in the hippocampus is affected by chronic psychosocial stress and concomitant fluoxetine treatment. Neuropsychopharmacology 31: 1616-1626.

Davidson JS, Baumgarten IM (1988). Glycyrrhetinic acid derivatives: a novel class of inhibitors of gap-junctional intercellular communication. Structure-activity relationships. J Pharmacol Exp Ther 246: 1104-1107.

DeBattista C, Belanoff J, Glass S, Khan A, Horne RL, Blasey C et al (2006). Mifepristone versus placebo in the treatment of psychosis in patients with psychotic major depression. Biol Psychiatry 60: 1343-1349.

Dermietzel R, Hertberg EL, Kessler JA, Spray DC (1991). Gap junctions between cultured astrocytes: immunocytochemical, molecular, and electrophysiological analysis. J Neurosci 11: 1421-1432.

Evans WH, Leybaert L (2007). Mimetic peptides as blockers of connexin channel-facilitated intercellular communication. Cell Commun Adhes 14: 265-273.

Fatemi SH, Folsom TD, Reutiman TJ, Pandian T, Braun NN, Haug K (2008). Chronic psychotropic drug treatment causes differential expression of connexin 43 and GFAP in frontal cortex of rats. Schizophr Res 104: 127-134.

Flores BH, Kenna H, Keller J, Solvason HB, Schatzberg AF (2006). Clinical and biological effects of mifepristone treatment for psychotic depression. Neuropsychopharmacology 31: 628-636.

Frantseva MV, Kokarovtseva L, Perez Velazquez JL (2002). Ischemia-induced brain damage depends on specific gapjunctional coupling. J Cereb Blood Flow Metab 22: 453-462.

Gajda Z, Gyengesi E, Hermesz E, Ali KS, Szente M (2003). Involvement of gap junctions in the manifestation and control of the duration of seizures in rats in vivo. Epilepsia 44: 1596-1600. Giaume C, Fromaget C, el A, Cordier J, Glowinski J, Gros D (1991). Gap junctions in cultured astrocytes: single-channel currents 
and characterization of channel-forming protein. Neuron 6: 133-143.

Giaume C, Koulakoff A, Roux L, Holcman D, Rouach N (2010). Astroglial networks: a step further in neuroglial and gliovascular interactions. Nat Rev Neurosci 11: 87-99.

Giaume C, Theis M (2010). Pharmacological and genetic approaches to study connexin-mediated channels in glial cells of the central nervous system. Brain Res Rev 63: 160-176.

Gosselin RD, Gibney S, O'Malley D, Dinan TG, Cryan JF (2009). Region specific decrease in glial fibrillary acidic protein immunoreactivity in the brain of a rat model of depression. Neuroscience 159: 915-925.

Harris AL (2007). Connexin channel permeability to cytoplasmic molecules. Prog Biophys Mol Biol 94: 120-143.

Herman JP, Adams D, Prewitt C (1995). Regulatory changes in neuroendocrine stress-integrative circuitry produced by a variable stress paradigm. Neuroendocrinology 61: 180-190.

Herrero-Gonzalez S, Valle-Casuso JC, Sanchez-Alvarez R, Giaume C, Medina JM, Tabernero A (2009). Connexin43 is involved in the effect of endothelin-1 on astrocyte proliferation and glucose uptake. Glia 57: 222-233.

Herve JC, Sarrouilhe D (2005). Connexin-made channels as pharmacological targets. Curr Pharm Des 11: 1941-1958.

Holsboer F, Barden N (1996). Antidepressants and hypothalamicpituitary-adrenocortical regulation. Endocr Rev 17: 187-205.

Hosseinzadeh H, Asl MN, Parvardeh S, Tagi Mansouri SM (2005). The effects of carbenoxolone on spatial learning in the Morris water maze task in rats. Med Sci Monit 11: BR88-BR94.

Jasinska M, Siucinska E, Glazewski S, Pyza E, Kossut M (2006). Characterization and plasticity of the double synapse spines in the barrel cortex of the mouse. Acta Neurobiol Exp (Wars) 66: 99-104.

Kimelberg HK (2007). Supportive or information-processing functions of the mature protoplasmic astrocyte in the mammalian CNS? A critical appraisal. Neuron Glia Biol 3: 181-189.

Kokarovtseva L, Jaciw-Zurakiwsky T, Mendizabal AR, Frantseva MV, Perez Velazquez JL (2009). Excitability and gap junctionmediated mechanisms in nucleus accumbens regulate selfstimulation reward in rats. Neuroscience 159: 1257-1263.

Kunzelmann P, Schroder W, Traub O, Steinhauser C, Dermietzel R, Willecke K (1999). Late onset and increasing expression of the gap junction protein connexin30 in adult murine brain and long-term cultured astrocytes. Glia 25: 111-119.

Lopez JF, Akil H, Watson SJ (1999). Neural circuits mediating stress. Biol Psychiatry 46: 1461-1471.

Madsen TM, Yeh DD, Valentine GW, Duman RS (2005). Electroconvulsive seizure treatment increases cell proliferation in rat frontal cortex. Neuropsychopharmacology 30: 27-34.

Miguel-Hidalgo J, Shoyama Y, Wanzo V (2009). Infusion of gliotoxins or a gap junction blocker in the prelimbic cortex increases alcohol preference in Wistar rats. $J$ Psychopharmacol 23: $550-557$.

Molteni R, Calabrese F, Cattaneo A, Mancini M, Gennarelli M, Racagni $G$ et al (2009). Acute stress responsiveness of the neurotrophin BDNF in the rat hippocampus is modulated by chronic treatment with the antidepressant duloxetine. Neuropsychopharmacology 34: 1523-1532.

Nagy JI, Rash JE (2000). Connexins and gap junctions of astrocytes and oligodendrocytes in the CNS. Brain Res Brain Res Rev 32: $29-44$.

Nilsen KE, Kelso AR, Cock HR (2006). Antiepileptic effect of gapjunction blockers in a rat model of refractory focal cortical epilepsy. Epilepsia 47: 1169-1175.

Ongur D, Drevets WC, Price JL (1998). Glial reduction in the subgenual prefrontal cortex in mood disorders. Proc Natl Acad Sci USA 95: 13290-13295.

Oomen CA, Mayer JL, de Kloet ER, Joels M, Lucassen PJ (2007). Brief treatment with the glucocorticoid receptor antagonist mifepristone normalizes the reduction in neurogenesis after chronic stress. Eur J Neurosci 26: 3395-3401.

Paxinos G, Watson C (1998). The Rat Brain in Stereotaxic Coordinates. Academic Press: San Diego.

Perea G, Navarrete M, Araque A (2009). Tripartite synapses: astrocytes process and control synaptic information. Trends Neurosci 32: 421-431.

Peters O, Schipke CG, Philipps A, Haas B, Pannasch U, Wang LP et al (2009). Astrocyte function is modified by Alzheimer's disease-like pathology in aged mice. J Alzheimers Dis 18: 177-189.

Rajkowska G (2000). Postmortem studies in mood disorders indicate altered numbers of neurons and glial cells. Biol Psychiatry 48: 766-777.

Rajkowska G, Miguel-Hidalgo JJ (2007). Gliogenesis and glial pathology in depression. CNS Neurol Disord Drug Targets 6: 219-233.

Rajkowska G, Miguel-Hidalgo JJ, Wei J, Dilley G, Pittman SD, Meltzer HY et al (1999). Morphometric evidence for neuronal and glial prefrontal cell pathology in major depression. Biol Psychiatry 45: 1085-1098.

Ross FM, Gwyn P, Spanswick D, Davies SN (2000). Carbenoxolone depresses spontaneous epileptiform activity in the CA1 region of rat hippocampal slices. Neuroscience 100: 789-796.

Rouach N, Koulakoff A, Abudara V, Willecke K, Giaume C (2008). Astroglial metabolic networks sustain hippocampal synaptic transmission. Science 322: 1551-1555.

Samarasinghe RA, Di MR, Volonte D, Galbiati F, Lewis M, Romero G et al (2011). Nongenomic glucocorticoid receptor action regulates gap junction intercellular communication and neural progenitor cell proliferation. Proc Natl Acad Sci USA 108: 16657-16662.

Samoilova M, Li J, Pelletier MR, Wentlandt K, Adamchik Y, Naus CC et al (2003). Epileptiform activity in hippocampal slice cultures exposed chronically to bicuculline: increased gap junctional function and expression. J Neurochem 86: 687-699.

Santarelli L, Gobbi G, Debs PC, Sibille ET, Blier P, Hen R et al (2001). Genetic and pharmacological disruption of neurokinin 1 receptor function decreases anxiety-related behaviors and increases serotonergic function. Proc Natl Acad Sci USA 98: 1912-1917.

Santarelli L, Saxe M, Gross C, Surget A, Battaglia F, Dulawa S et al (2003). Requirement of hippocampal neurogenesis for the behavioral effects of antidepressants. Science 301: 805-809.

Scemes E, Giaume C (2006). Astrocyte calcium waves: what they are and what they do. Glia 54: 716-725.

Simpson GM, El SA, Loza N, Kingsbury SJ, Fayek M, Rady A et al (2005). An 8-week open-label trial of a 6-day course of mifepristone for the treatment of psychotic depression. J Clin Psychiatry 66: 598-602.

Theis M, Jauch R, Zhuo L, Speidel D, Wallraff A, Doring B et al (2003). Accelerated hippocampal spreading depression and enhanced locomotory activity in mice with astrocyte-directed inactivation of connexin43. J Neurosci 23: 766-776.

Theis M, Sohl G, Eiberger J, Willecke K (2005). Emerging complexities in identity and function of glial connexins. Trends Neurosci 28: 188-195.

Wallraff A, Kohling R, Heinemann U, Theis M, Willecke K, Steinhauser C (2006). The impact of astrocytic gap junctional coupling on potassium buffering in the hippocampus. J Neurosci 26: 5438-5447.

Warner-Schmidt JL, Duman RS (2007). VEGF is an essential mediator of the neurogenic and behavioral actions of antidepressants. Proc Natl Acad Sci USA 104: 4647-4652.

Willner P (1997). Validity, reliability and utility of the chronic mild stress model of depression: a 10-year review and evaluation. Psychopharmacology (Berl) 134: 319-329. 
Willner P (2005). Chronic mild stress (CMS) revisited: consistency and behavioural-neurobiological concordance in the effects of CMS. Neuropsychobiology 52: 90-110.

Willner P, Muscat R, Papp M (1992). Chronic mild stress-induced anhedonia: a realistic animal model of depression. Neurosci Biobehav Rev 16: 525-534.

Willner P, Towell A, Sampson D, Sophokleous S, Muscat R (1987). Reduction of sucrose preference by chronic unpredictable mild stress, and its restoration by a tricyclic antidepressant. Psychopharmacology (Berl) 93: 358-364.

Wu LM, Han H, Wang QN, Hou HL, Tong H, Yan XB et al (2007). Mifepristone repairs region-dependent alteration of synapsin I in hippocampus in rat model of depression. Neuropsychopharmacology 32: 2500-2510.

Xia X, Batra N, Shi Q, Bonewald LF, Sprague E, Jiang JX (2010). Prostaglandin promotion of osteocyte gap junction function through transcriptional regulation of connexin 43 by glycogen synthase kinase 3/beta-catenin signaling. Mol Cell Biol 30: 206-219.

Xie M, Yi C, Luo X, Xu S, Yu Z, Tang Y et al (2011). Glial gap junctional communication involvement in hippocampal damage after middle cerebral artery occlusion. Ann Neurol 70: 121-132.

Zhang Y, Gu F, Chen J, Dong W (2010). Chronic antidepressant administration alleviates frontal and hippocampal BDNF deficits in CUMS rat. Brain Res 1366: 141-148.

Supplementary Information accompanies the paper on the Neuropsychopharmacology website (http://www.nature.com/npp) 